\title{
ANALYTIC HYPOELLIPTICITY AND LOCAL SOLVA- BILITY FOR A CLASS OF PSEUDO-DIFFERENTIAL OPERATORS WITH SYMPLECTIC CHARACTERISTICS
}

\author{
TSUTOMU SAKURAI \\ Department of Mathematics, Saitama University \\ 255 Shimo-okubo, Urawa 338, Japan \\ E-mail: tsakurai@rimath.saitama-u.ac.jp
}

1. Introduction. Let us consider a classical analytic pseudo-differential operator $P$ of order $\mu$ on an open set $\Omega$ in $\mathbf{R}^{N}$ with the symbol

$$
p(x, \xi) \sim p_{\mu}(x, \xi)+p_{\mu-1}(x, \xi)+\ldots,
$$

where $p_{\mu-j}(x, \xi)$ is positively homogeneous of degree $\mu-j$ with respect to $\xi$. We assume that the characteristic set $\Sigma=p_{\mu}^{-1}(0)$ of $P$ is a symplectic real analytic submanifold of $T^{*}(\Omega) \backslash 0$ of codimension $2 d$ and that $p_{\mu}$ vanishes exactly at the order $m$ on $\Sigma$. As in Grušin [4], Sjöstrand [11] and Métivier [8], we also assume that $p_{\mu-j}$ vanishes at the order $m-2 j$ on $\Sigma$ for $j \leq m / 2$.

$C^{\infty}$ and analytic hypoellipticity of this class of operators has been extensively studied by many mathematicians (see e.g., [1], [2], [4], [8], [9], [11], [13] and others). Among them Métivier [8] has proved analytic hypoellipticity of $P$ by constructing a left parametrix when $P$ is subelliptic with loss of $m / 2$ derivatives.

In this note, we study hypoellipticity and local solvability of $P$ at a point where the above subellipticity condition is not satisfied. We shall then construct a system of analytic pseudo-differential operators on $\mathbf{R}^{N-d}$ to which we can reduce the study of analytic hypoellipticity and local solvability of $P$.

Typical examples of the operators are

$$
P=D_{1}^{2}+x_{1}^{2} D_{2}^{2}-\left(1+x_{1}^{k}\right) D_{2}, \quad \text { in } \mathbf{R}^{2}
$$

with $k \in \mathbf{N}$,

$$
P=D_{1}^{2}+x_{1}^{2}\left(D_{2}^{2}+D_{3}^{2}\right)-\left(1-x_{2}^{2}\right) D_{3}-c, \quad \text { in } \mathbf{R}^{3}
$$

1991 Mathematics Subject Classification: Primary 35H05.

The paper is in final form and no version of it will be published elsewhere. 
with $c \in \mathbf{C}$. We can show that the operators (1.1) and (1.2) are analytic hypoelliptic and locally solvable for all $k$ and all $c$ respectively.

\section{Notation and statement of the main result}

2.1. Notation. Let $\Omega$ be an open set in $\mathbf{R}^{N}$. We denote by $x^{*}=(x, \xi)$ a point in $T^{*}(\Omega) \backslash 0$. For a distribution $u \in \mathcal{D}^{\prime}(\Omega), W F_{A}(u)$ is the analytic wave front set of $u$. We introduce the presheaf $\mathcal{C}_{\Omega}^{f}$ of micro-distributions on $\Omega$ as follows: With each open set $\omega \subset T^{*}(\Omega) \backslash 0$ we associate the space

$$
\mathcal{C}_{\Omega}^{f}(\omega)=\mathcal{D}^{\prime}(\Omega) /\left\{u \in \mathcal{D}^{\prime}(\Omega) ; W F_{A}(u) \cap \omega=\emptyset\right\} .
$$

We shall also use the notation:

$$
\begin{aligned}
& \mathcal{A}_{\Omega}\left(\stackrel{\circ}{x}^{*}\right)=\left\{u \in \mathcal{D}^{\prime}(\Omega) ; \stackrel{\triangleright}{x}^{*} \notin W F_{A}(u)\right\}, \\
& \mathcal{C}_{\Omega}^{f}\left(\stackrel{\circ}{x}^{*}\right)=\underset{\overrightarrow{\omega \ni \AA^{*}}}{\lim } \mathcal{C}_{\Omega}^{f}(\omega)=\mathcal{D}^{\prime}(\Omega) / \mathcal{A}_{\Omega}\left(\stackrel{\circ}{x}^{*}\right)
\end{aligned}
$$

for $\stackrel{\circ}{x}^{*} \in T^{*}(\Omega) \backslash 0$, for the space of distributions on $\Omega$ which are micro-analytic at $\stackrel{\circ}{x}^{*}$ and for the space of germs at $\stackrel{\circ}{x}^{*}$ of micro-distributions on $\Omega$ respectively.

Let $\Omega \times \Gamma$ be a conic neighborhood of a point $(\stackrel{\circ}{x}, \stackrel{\circ}{\theta})$ in $\mathbf{R}^{N} \times\left(\mathbf{R}^{n} \backslash 0\right)$. Let $\mu \in \mathbf{R}$ and $h$ be the reciprocal of a positive integer. A formal sum $\sum_{j=0}^{\infty} a_{j}(x, \theta)$ will be called a polyhomogeneous analytic symbol on $\Omega \times \Gamma$ of degree $\mu$ and step $h$ if $a_{j}(x, \theta)$ is a holomorphic function on $\widetilde{\Omega} \times \widetilde{\Gamma}$, positively homogeneous of degree $\mu-j h$ with respect to $\theta$ and satisfying the estimate

$$
\left|a_{j}(x, \theta)\right| \leq C^{j+1}(j !)^{h}|\theta|^{m-j h}
$$

for all $(x, \theta) \in \widetilde{\Omega} \times \widetilde{\Gamma}$ with $C$ independent of $j$, where $\widetilde{\Omega}$ is a complex neighborhood of $\Omega$ in $\mathbf{C}^{N}$ and $\widetilde{\Gamma}$ is a conic complex neighborhood of $\Gamma$ in $\mathbf{C}^{n} \backslash 0$. Then we shall write $\sum_{j=0}^{\infty} a_{j}(x, \theta) \in a-S_{\text {phg }}^{\mu, h}(\Omega \times \Gamma)$.

Let us also recall the definition of analytic symbols of type $(\rho, \delta)$ introduced by Métivier [8]: For $\rho \in(0,1], \delta \in[0,1)$ and a conic set $\Omega \times \Gamma \subset \mathbf{R}^{N} \times\left(\mathbf{R}^{n} \backslash 0\right)$, the space $a-S_{\rho, \delta}(\Omega \times \Gamma)$ of analytic symbols on $\Omega \times \Gamma$ of degree $\mu$ and type $(\rho, \delta)$ is the set of $C^{\infty}$ functions $a(x, \theta)$ on $\Omega \times \Gamma$ for which there are $C>0$ and $R>0$ such that

$$
\left|\partial_{x}^{\alpha} \partial_{\theta}^{\beta} a(x, \theta)\right| \leq C^{|\alpha|+|\beta|+1}(1+|\theta|)^{\mu}\left(|\alpha|+|\alpha|^{1-\delta}|\theta|^{\delta}\right)^{|\alpha|}(|\beta| /|\theta|)^{\rho|\beta|}
$$

for all multi-indices $\alpha, \beta$ and all $(x, \theta) \in \Omega \times \Gamma$ such that $R|\beta| \leq|\theta|$. Moreover, a symbol $a \in a-S_{\rho, \delta}^{\mu}(\Omega \times \Gamma)$ is said to be equivalent to $0(a \sim 0)$ in $\Omega_{0} \times \Gamma_{0} \subset \Omega \times \Gamma$ if there is a constant $\varepsilon>0$ such that

$$
\left|\partial_{x}^{\alpha} a(x, \theta)\right| \leq(1 / \varepsilon)^{|\alpha|+1} e^{-\varepsilon|\theta|}
$$

for all multi-indices $\alpha$ and all $(x, \theta) \in \Omega_{0} \times \Gamma_{0}$.

Each polyhomogeneous symbol has a realization in $a-S_{1,0}^{\mu}(\Omega \times \Gamma)$ as follows: Let $\left\{\chi_{j}(\theta)\right\}_{j=0}^{\infty}$ be a sequence in $C^{\infty}\left(\mathbf{R}^{n}\right)$ such that $\chi_{j}(\theta)=0$ for $|\theta| \leq j, \chi_{j}(\theta)=1$ for $|\theta| \geq 2 j$ and there is a constant $C>0$ for which we have $\left|\partial_{\theta}^{\alpha} \chi_{j}(\theta)\right| \leq C^{|\alpha|}$ for all $j, \alpha$ such that $|\alpha| \leq j$. If $\sum_{j=0}^{\infty} a_{j} \in a-S_{\text {phg }}^{\mu, h}(\Omega \times \Gamma)$ then, for $\lambda>0$ large enough,

$$
a(x, \theta)=\sum_{j=0}^{\infty} \chi_{j+1}(\theta / \lambda) a_{j}(x, \theta)
$$


is in $a-S_{1,0}^{\mu}(\Omega \times \Gamma)$. (See e.g. Treves [14, Chap. V] or Métivier [M, Section III].) Any symbol $a \in a-S_{\rho, \delta}^{\mu}(\Omega \times \Gamma)$ which is equivalent to the symbol (2.1) will be called a realization of $\sum_{j=0}^{\infty} a_{j}$ and we shall then write $a \sim \sum_{j=0}^{\infty} a_{j}$. Also, we let $\sigma_{\mu}(a)(x, \theta)=a_{0}(x, \theta)$ denote the principal symbol of $a$.

If $\stackrel{\circ}{x}^{*}=(\stackrel{\circ}{x}, \stackrel{\circ}{\xi}) \in \Omega \times \Gamma \subset T^{*}\left(\mathbf{R}^{N}\right) \backslash 0$ and $a(x, \xi) \in a-S_{\rho, \delta}^{\mu}(\Omega \times \Gamma)$, then we define the operator

$$
\operatorname{op}(a)_{\stackrel{\circ}{*}^{*}}: \mathcal{C}_{\Omega}^{f}\left(\stackrel{\circ}{x}^{*}\right) \rightarrow \mathcal{C}_{\Omega}^{f}\left(\stackrel{\circ}{x}^{*}\right)
$$

via the distribution kernel

$$
A_{x^{*}}(x, y)=\phi(x)\left((2 \pi)^{-N} \int_{\mathbf{R}^{N}} e^{i(x-y) \xi} a(x, \xi) g(\xi) d \xi\right) \phi(y),
$$

where $\phi \in C_{0}^{\infty}(\Omega), \phi(x)=1$ in a neighborhood of $\stackrel{\circ}{x}$ and $g(\xi) \in C^{\infty}\left(\mathbf{R}^{N}\right)$ is a cut-off function introduced in Lemma 3.1 of Métivier [8] such that $\operatorname{supp}(g) \subset \Gamma, g(\xi)=1$ in a conic neighborhood of $\stackrel{\circ}{\xi}$ for $|\xi| \geq 2$ and there are $C>0, \rho^{\prime} \in(0,1)$ for which we have

$$
\left|\partial_{\xi}^{\alpha} g(\xi)\right| \leq C^{|\alpha|+1}(|\alpha| /|\xi|)^{\rho^{\prime}|\alpha|}
$$

for all $\alpha, \xi$ such that $|\alpha| \leq|\xi|$.

The operator $\operatorname{op}(a)_{x^{*}}$ is well defined; that is, independent of the choice of the cut-off functions $\phi$ and $g$ in (2.2). Moreover, when $a(x, \xi)$ is a realization of a formal symbol $\sum_{j=0}^{\infty} a_{j}(x, \xi), \operatorname{op}(a)_{x^{*}}$ is also independent of the choice of the realization. Then $a\left(x, D_{x}\right)=\mathrm{op}(a)$ which stands for $\bigsqcup_{x^{*} \in \Omega \times \Gamma} \mathrm{op}(a)_{\hat{x}^{*}}$ is called an analytic pseudo-differential operator on $\Omega \times \Gamma$ with the symbol $a(x, \xi)$ (or $\sum_{j=0}^{\infty} a_{j}(x, \xi)$ ).

2.2. Statement of the result. Let $\Sigma$ be a symplectic submanifold of codimension $2 d$ in a conic set $\omega \subset T^{*}\left(\mathbf{R}^{N}\right) \backslash 0$. We consider a classical analytic pseudo-differential operator $P$ of order $\mu$ whose symbol $p(x, \xi) \sim \sum_{j=0}^{\infty} p_{\mu-j}(x, \xi)$ defined on $\omega$ is such that $p_{\mu-j}$ is homogeneous of degree $\mu-j$, and vanishes to order $m-2 j$ on $\Sigma$ for $j \leq m / 2$.

After transforming $P$ by a suitable elliptic Fourier integral operator, we may suppose $\Sigma$ is given by the equation

$$
x_{1}=\ldots=x_{d}=0 ; \quad \xi_{1}=\ldots=\xi_{d}=0 .
$$

Henceforth, we write $t_{i}=x_{i}, \tau_{i}=\xi_{i}$ for $i=1, \ldots, d$ and $y_{i}=x_{d+i}, \eta_{i}=\xi_{d+i}$ for $i=1, \ldots, n(=N-d)$ and set

$$
\iota: T^{*}\left(\mathbf{R}^{n}\right) \backslash 0 \ni(y, \eta) \mapsto(0, y, 0, \eta) \in T^{*}\left(\mathbf{R}^{N}\right) \backslash 0 .
$$

In this coordinate, $\Sigma$ can be identified with $\iota\left(T^{*}\left(\mathbf{R}^{n}\right) \backslash 0\right)$ in $\omega$ and $P$ has the form

$$
P=\sum_{|\alpha|+|\beta| \leq m} t^{\alpha} c_{\alpha \beta}\left(x, D_{x}\right) D_{t}^{\beta}, \quad c_{\alpha \beta} \in a-S_{\mathrm{phg}}^{\mu-m / 2+|\alpha| / 2-|\beta| / 2,1 / 2}(\omega) .
$$

For $\stackrel{\circ}{x}^{*}=\iota\left(\stackrel{\circ}{y}^{*}\right)=(0, \stackrel{\circ}{y}, 0, \stackrel{\circ}{\eta}) \in \Sigma \cap \omega$, we set

$$
\begin{aligned}
\sigma_{\Sigma}^{0}(P)_{\grave{x}^{*}}(t, \tau) & =\sum_{|\alpha|+|\beta|=m} \sigma_{\mu-m / 2+|\alpha| / 2-|\beta| / 2}\left(c_{\alpha \beta}\right)\left(\stackrel{\circ}{x}^{*}\right) t^{\alpha} \tau^{\beta}, \\
\widehat{\sigma}_{\Sigma}(P)_{\stackrel{x}{*}^{*}}\left(t, D_{t}\right) & =\sum_{|\alpha|+|\beta| \leq m} \sigma_{\mu-m / 2+|\alpha| / 2-|\beta| / 2}\left(c_{\alpha \beta}\right)\left(\stackrel{\circ}{x}^{*}\right) t^{\alpha} D_{t}^{\beta}
\end{aligned}
$$


and assume

$$
\exists C>0 \text { such that }\left|\sigma_{\Sigma}^{0}(P)_{\stackrel{\circ}{*}^{*}}(t, \tau)\right| \geq C(|t|+|\tau|)^{m} .
$$

With this assumption $\widehat{\sigma}_{\Sigma}(P)_{\grave{x}^{*}}$ becomes a Fredholm operator from $\mathcal{S}^{\prime}$ to $\mathcal{S}^{\prime}$, and if $\operatorname{Ker}\left(\widehat{\sigma}_{\Sigma}(P)_{\dot{x}^{*}}\right) \cap \mathcal{S}=\{0\}$ (resp. $\left.\operatorname{Coker}\left(\widehat{\sigma}_{\Sigma}(P)_{{ }_{x^{*}}}\right) \cap \mathcal{S}=\{0\}\right)$ then $P$ (resp. $P^{*}$ ) is subelliptic with loss of $m / 2$ derivatives. Our interest is now focusing at a point where this subellipticity condition of $P$ or $P^{*}$ is not satisfied. So we set

$$
k_{+}=\operatorname{dim}\left(\operatorname{Ker}\left(\widehat{\sigma}_{\Sigma}(P)_{\stackrel{\circ}{x}^{*}}\right) \cap \mathcal{S}\right), \quad k_{-}=\operatorname{dim}\left(\operatorname{Coker}\left(\widehat{\sigma}_{\Sigma}(P)_{\stackrel{\circ}{x}^{*}}\right) \cap \mathcal{S}\right) .
$$

The main theorem of this note is

TheOREm 2.1. Let $P$ be an operator of the form (2.4) satisfying (2.5). Then there exist a $k_{-} \times k_{+}$-matrix of pseudo-differential operators

$$
M\left(y, D_{y}\right):\left(\mathcal{C}_{\mathbf{R}^{n}}^{f}\left(\stackrel{\circ}{ }^{*}\right)\right)^{k_{+}} \rightarrow\left(\mathcal{C}_{\mathbf{R}^{n}}^{f}\left(\stackrel{\circ}{y}^{*}\right)\right)^{k_{-}}
$$

and two operators

$$
H^{+}:\left(\mathcal{C}_{\mathbf{R}^{n}}^{f}\left(\stackrel{\circ}{y}^{*}\right)\right)^{k_{+}} \rightarrow \mathcal{C}_{\mathbf{R}^{N}}^{f}\left(\stackrel{\diamond}{x}^{*}\right) \quad \text { and } \quad H^{-*}: \mathcal{C}_{\mathbf{R}^{N}}^{f}\left(\stackrel{\circ}{x}^{*}\right) \rightarrow\left(\mathcal{C}_{\mathbf{R}^{n}}^{f}\left(\stackrel{\circ}{ }^{*}\right)\right)^{k_{-}}
$$

for which we have the isomorphisms:

$$
\begin{aligned}
& H^{+}: \operatorname{Ker}\left(M:\left(\mathcal{C}_{\mathbf{R}^{n}}^{f}\left(\stackrel{\circ}{y}^{*}\right)\right)^{k_{+}} \rightarrow\left(\mathcal{C}_{\mathbf{R}^{n}}^{f}\left(\stackrel{\circ}{y}^{*}\right)\right)^{k_{-}}\right) \\
& \stackrel{\sim}{\rightarrow} \operatorname{Ker}\left(P: \mathcal{C}_{\mathbf{R}^{N}}^{f}\left(\stackrel{\leftrightarrow}{x}^{*}\right) \rightarrow \mathcal{C}_{\mathbf{R}^{N}}^{f}\left(\stackrel{\leftrightarrow}{x}^{*}\right)\right) \\
& H^{-*}: \operatorname{Coker}\left(P: \mathcal{C}_{\mathbf{R}^{N}}^{f}\left(\stackrel{\circledR}{x}^{*}\right) \rightarrow \mathcal{C}_{\mathbf{R}^{N}}^{f}\left(\stackrel{\leftrightarrow}{x}^{*}\right)\right) \\
& \stackrel{\sim}{\rightarrow} \operatorname{Coker}\left(M:\left(\mathcal{C}_{\mathbf{R}^{n}}^{f}\left(\stackrel{\circ}{y}^{*}\right)\right)^{k_{+}} \rightarrow\left(\mathcal{C}_{\mathbf{R}^{n}}^{f}\left(\stackrel{\circ}{y}^{*}\right)\right)^{k_{-}}\right) .
\end{aligned}
$$

Remark. Grigis-Rothschild [3] have treated the case $c_{\alpha \beta}=c_{\alpha \beta}\left(D_{y}\right)$ and obtained the same result as above. See also Kashiwara-Kawai-Oshima [7] and Stein [12].

\section{Operator valued symbols}

3.1. Symbol spaces. Let $\stackrel{\circ}{y}^{*}=(\stackrel{\circ}{y}, \stackrel{\circ}{\eta}) \in \mathbf{R}^{n} \times\left(\mathbf{R}^{n} \backslash 0\right)(|\stackrel{\eta}{\eta}|=1)$. For $\rho>0$, we consider a complex neighborhood of $\stackrel{\circ}{ }^{*}$ of the form

$$
\omega_{\rho}=\left\{(y, \eta) \in \mathbf{C}^{n} \times\left(\mathbf{C}^{n} \backslash 0\right) ;|y-\stackrel{o}{y}|<\rho,|\eta-\stackrel{\circ}{\eta}|<\rho\right\}
$$

and let $\widetilde{\omega}_{\rho}$ denote the cone generated by $\omega_{\rho}$; that is,

$$
\widetilde{\omega}_{\rho}=\left\{(y, \eta) \in \mathbf{C}^{n} \times\left(\mathbf{C}^{n} \backslash 0\right) ;|y-\stackrel{\circ}{y}|<\rho,|\eta /| \eta|-\stackrel{\imath}{\eta}|<\rho\right\} .
$$

Let $B=B(\lambda)$ be some Banach space whose norm may depend on $\lambda$.

Definition 3.1. Let $\mu \in \mathbf{R}$. The space $\mathcal{O}^{(\mu)}\left(\widetilde{\omega}_{\rho} ; B\right)$ of $B$-valued homogeneous symbols (also denoted by $B_{\rho}^{(\mu)}$ for short) and the space $S_{\mathrm{phg}}^{\mu, h}\left(\widetilde{\omega}_{\rho} ; B\right)$ of B-valued polyhomogeneous symbols are defined by:

(1) $p(y, \eta) \in \mathcal{O}^{(\mu)}\left(\widetilde{\omega}_{\rho} ; B\right)$ if and only if $p(y, \eta)$ is a holomorphic function defined on $\widetilde{\omega}_{\rho}$ with values in $B(|\eta|)$ which satisfies

$$
\|p(y, \lambda \eta)\|_{B(\lambda)}=\lambda^{\mu}\|p(y, \eta)\|_{B(1)} \quad \text { for }(y, \eta) \in \omega_{\rho}
$$

and

$$
\|p\|_{B_{\rho}^{(\mu)}} \stackrel{\text { def }}{=} \sup _{(y, \eta) \in \omega_{\rho}}\|p(y, \eta)\|_{B(1)}<+\infty .
$$


(2) $\sum_{j=0}^{\infty} p_{j}(y, \eta) \in S_{\text {phg }}^{\mu, h}\left(\widetilde{\omega}_{\rho} ; B\right)$ if and only if $p_{j}(y, \eta) \in \mathcal{O}^{(\mu-j h)}\left(\widetilde{\omega}_{\rho} ; B\right)$ and there exists a $C>0$ such that

$$
\left\|p_{j}\right\|_{B_{\rho}^{(\mu-j h)}} \leq C^{j+1}(j !)^{h}
$$

3.2. Banach spaces and estimates. Let us now introduce several Banach spaces following Métivier [8] and quote some of their properties from [8].

Definition 3.2. $\mathcal{A}^{m}(\lambda)$ denotes the space of differential operators on $\mathbf{R}^{d}$ of the form

$$
A\left(t, D_{t}\right)=\sum_{|\alpha|+|\beta| \leq m} C_{\alpha \beta} t^{\alpha} D_{t}^{\beta}, \quad C_{\alpha \beta} \in \mathbf{C},
$$

with the norm $\|A\|_{\mathcal{A}^{m}(\lambda)}=\sum_{\alpha, \beta}\left|C_{\alpha \beta}\right| \lambda^{(|\beta|-|\alpha|) / 2}$.

Definition 3.3. $\mathcal{M}^{ \pm}$denotes the space of $k_{-} \times k_{+}$-matrices $M=\left(m_{i j}\right) \in L\left(\mathbf{C}^{k_{+}}, \mathbf{C}^{k_{-}}\right)$ with the norm $\|M\|_{\mathcal{M}^{ \pm}(\lambda)}=\left(\sum\left|m_{i j}\right|^{2}\right)^{1 / 2}$ independent of $\lambda$.

Let $t$ denote a point in $\mathbf{R}^{d}$. We consider the operators

$$
T_{j}=T_{j}(\lambda)=\lambda^{-\frac{1}{2}} \frac{\partial}{\partial t_{j}}, \quad T_{-j}=T_{-j}(\lambda)=i \lambda^{1 / 2} t_{j}, \quad j=1, \ldots, d .
$$

For a sequence $I=\left(j_{1}, \ldots, j_{k}\right) \in\{ \pm 1, \ldots, \pm d\}^{k}$ we write $|I|=k$ and $T_{I}=T_{j_{1}}, \ldots, T_{j_{k}}$. If $L$ is an operator acting from $\mathcal{S}\left(\mathbf{R}^{d}\right)$ to $\mathcal{S}^{\prime}\left(\mathbf{R}^{d}\right)$ we write

$$
\left(\operatorname{ad} T_{j}\right)(L)=\left[T_{j}, L\right]=T_{j} L-L T_{j}
$$

and because the ad $T_{j}$ 's commute, we write for a multi-index $\alpha=\left(\alpha_{j}\right)_{j= \pm 1, \ldots, \pm d} \in \mathbf{N}^{2 d}$,

$$
(\operatorname{ad} T)^{\alpha}=\prod_{j}\left(\operatorname{ad} T_{j}\right)^{\alpha_{j}} .
$$

Also we write $\|L\|_{0}$ for the operator-norm of $L$ from $L^{2}\left(\mathbf{R}^{d}\right)$ to $L^{2}\left(\mathbf{R}^{d}\right)$.

Definition 3.4. Let $m$ be a non-negative integer. For a real $R>0, \mathcal{L}_{R}^{m}(\lambda)$ denotes the space of the operators for which there is a constant $C$ such that for all multi-indices $\alpha \in \mathbf{N}^{2 d}$ and for all $I, J$ with $|I|+|J| \leq|\alpha|+m$,

$$
\left\|T_{I}\left(\operatorname{ad} T_{j}\right)^{\alpha}(L) T_{J}\right\|_{0} \leq C|\alpha| ! R^{|\alpha|} .
$$

Clearly $\mathcal{L}_{R}^{m}(\lambda)$ becomes a Banach space and there exists $C>0$ such that

$$
\|A L\|_{\mathcal{L}_{R}^{0}(\lambda)} \leq C\|A\|_{\mathcal{A}^{m}(\lambda)}\|L\|_{\mathcal{L}_{R}^{m}(\lambda)}
$$

for all $A \in \mathcal{A}^{m}(\lambda)$ and $L \in \mathcal{L}_{R}^{m}(\lambda)$.

For an operator $K$ from $\mathcal{S}\left(\mathbf{R}^{d}\right)$ to $\mathcal{S}^{\prime}\left(\mathbf{R}^{d}\right)$ we write $K(t, s)$ for its distribution kernel. We also introduce the operator $\widetilde{K}$ induced from $K$ via the Fourier transform; that is,

$$
\widetilde{K} \widehat{u}=\widehat{K u} .
$$

Definition 3.5. For $\varepsilon>0, \mathcal{B}_{\varepsilon}(\lambda)$ is the space of Hilbert-Schmidt operators $K$ such that for all $j=1, \ldots, d$,

$$
\begin{aligned}
\left\|e^{\varepsilon \lambda \phi_{j}(t, s)} K(t, s)\right\|_{L^{2}\left(\mathbf{R}^{d} \times \mathbf{R}^{d}\right)} & <+\infty, \\
\left\|e^{\varepsilon \phi_{j}(\tau, \sigma) / \lambda} \widetilde{K}(\tau, \sigma)\right\|_{L^{2}\left(\mathbf{R}^{d} \times \mathbf{R}^{d}\right)} & <+\infty,
\end{aligned}
$$


where $\phi_{j}(t, s)=\left|t_{j}\right| t_{j}\left|-s_{j}\right| s_{j}||$. The norm of $\mathcal{B}_{\varepsilon}(\lambda)$ is the maximum for $j=1, \ldots, d$ of the norms in (3.2) and (3.3).

The space $\mathcal{B}_{\varepsilon}(\lambda)$ plays an important role in the construction of a relative parametrix. The crucial points are

Lemma 3.6 (Métivier [8], Proposition 2.8). If $m>d$ then for all $R>0$ there exist $\varepsilon>0$ and $C$ such that

for all $K \in \mathcal{L}_{R}^{m}(\lambda)$.

$$
\|K\|_{\mathcal{B}_{\varepsilon}(\lambda)} \leq C\|K\|_{\mathcal{L}_{R}^{m}(\lambda)}
$$

Lemma 3.7 (loc. cit., Proposition 2.9). For all $R>0$, there exist $\varepsilon_{0}>0$ and $C$ such that for all $\varepsilon \in\left(0, \varepsilon_{0}\right]$,

$$
\|L K\|_{\mathcal{B}_{\varepsilon}(\lambda)} \leq C\|L\|_{\mathcal{L}_{R}^{0}(\lambda)}\|K\|_{\mathcal{B}_{\varepsilon}(\lambda)}
$$

for all $L \in \mathcal{L}_{R}^{0}(\lambda)$ and all $K \in \mathcal{B}_{\varepsilon}(\lambda)$.

Lemma 3.8 (loc. cit., Proposition 2.10). There exists a constant $M_{0}$ such that for all $0<\varepsilon^{\prime}<\varepsilon \leq 1$ and all $j= \pm 1, \ldots, \pm d$,

$$
\left\|\left(\operatorname{ad} T_{j}\right)(K)\right\|_{\mathcal{B}_{\varepsilon^{\prime}}(\lambda)} \leq\left(\frac{M_{0}}{\varepsilon-\varepsilon^{\prime}}\right)^{1 / 2}\|K\|_{\mathcal{B}_{\varepsilon}(\lambda)}
$$

for all $K \in \mathcal{B}_{\varepsilon}(\lambda)$.

For the operator $K$ of kernel $K(t, s)$, we define its symbol $k=\sigma(K)$ by

$$
k(t, \tau)=\int_{\mathbf{R}^{d}} K(t, t-s) e^{-i s \tau} d s
$$

Then

$$
K u(t)=k\left(t, D_{t}\right) u(t)=(2 \pi)^{-d} \int_{\mathbf{R}^{d}} e^{i t \tau} k(t, \tau) \widehat{u}(\tau) d \tau .
$$

LEMma 3.9. For all $\varepsilon>0$, there exists a $C>0$ such that for all $(\alpha, \beta) \in \mathbf{R}^{d} \times \mathbf{R}^{d}$,

$$
\sup _{(t, \tau) \in \mathbf{R}^{2 d}}\left|\partial_{t}^{\alpha} \partial_{\tau}^{\beta} \sigma(K)(t, \tau)\right| \leq C^{j+1}(|\alpha|+|\beta|)^{(|\alpha|+|\beta|) / 2} \lambda^{(|\alpha|-|\beta|) / 2}\|K\|_{\mathcal{B}_{\varepsilon}(\lambda)}
$$

for all $K \in \mathcal{B}_{\varepsilon}(\lambda)$.

We also introduce the space of Hermite operators. First we define its symbol space.

Definition 3.10. For $\varepsilon>0, \mathcal{H}_{\varepsilon}(\lambda)$ is the space of functions $h(t) \in \mathcal{S}\left(\mathbf{R}^{d}\right)$ such that for all $j=1, \ldots, d$,

$$
\begin{aligned}
\left\|e^{\lambda \varepsilon t_{j}^{2}} h(t)\right\|_{L^{2}\left(\mathbf{R}^{d}\right)} & <+\infty, \\
\left\|e^{\varepsilon \tau_{j}^{2} / \lambda} \widehat{h}(\tau)\right\|_{L^{2}\left(\mathbf{R}^{d}\right)} & <+\infty .
\end{aligned}
$$

The norm of $\mathcal{H}_{\varepsilon}(\lambda)$ is the maximum for $j=1, \ldots, d$ of the norms in (3.4) and (3.5).

For $H=\left(h_{1}, \ldots, h_{k}\right) \in\left(\mathcal{H}_{\varepsilon}(\lambda)\right)^{k}$, define the operators $H$ and $H^{*}$ by

$$
H: \mathbf{C}^{k} \ni\left(z_{l}\right)_{l=1}^{k} \mapsto \sum_{l=1}^{k} z_{l} h_{l}(t) \in \mathcal{S}\left(\mathbf{R}^{d}\right),
$$




$$
H^{*}: \mathcal{S}^{\prime}\left(\mathbf{R}^{d}\right) \ni u(t) \mapsto\left(\int_{\mathbf{R}^{d}} \overline{h_{l}(t)} u(t) d t\right)_{l=1}^{k} \in \mathbf{C}^{k},
$$

where $\overline{h_{l}(t)}$ is the complex conjugate of $h_{l}(t)$. We denote by $\mathcal{H}_{\varepsilon}^{k}(\lambda)$ and $\mathcal{H}_{\varepsilon}^{k *}(\lambda)$ the spaces of operators of the form (3.6) and (3.7) respectively. The norm in them is defined by

$$
\|H\|_{\mathcal{H}_{\varepsilon}^{k}(\lambda)}=\left\|H^{*}\right\|_{\mathcal{H}_{\varepsilon}^{k *}(\lambda)}=\left(\sum_{l=1}^{k}\left\|h_{l}\right\|_{\mathcal{H}_{\varepsilon}(\lambda)}^{2}\right)^{1 / 2}
$$

and we write $\sigma(H)=\sigma\left(H^{*}\right)=\left(h_{1}, \ldots, h_{k}\right)$.

By definition, we have

Lemma 3.11. Let $k, k^{\prime} \in \mathbf{N}$ and $\varepsilon>0$. If $K \in \mathcal{B}_{\varepsilon}(\lambda), H_{1}, H_{2} \in \mathcal{H}_{\varepsilon}^{k}(\lambda)$ and $H_{3} \in \mathcal{H}_{\varepsilon}^{k^{\prime}}(\lambda)$ then $K H_{1} \in \mathcal{B}_{\varepsilon}(\lambda), H_{2} H_{1}^{*} \in \mathcal{B}_{\varepsilon}(\lambda)$ and $H_{1}^{*} H_{3} \in L\left(\mathbf{C}^{k^{\prime}}, \mathbf{C}^{k}\right)$. Moreover,

$$
\begin{aligned}
& \left\|K H_{1}\right\|_{\mathcal{H}_{\varepsilon}^{k}(\lambda)} \leq\|K\|_{\mathcal{B}_{\varepsilon}(\lambda)}\left\|H_{1}\right\|_{\mathcal{H}_{\varepsilon}^{k}(\lambda)}, \\
& \left\|H_{2} H_{1}^{*}\right\|_{\mathcal{B}_{\varepsilon}(\lambda)} \leq\left\|H_{2}\right\|_{\mathcal{H}_{\varepsilon}^{k}(\lambda)}\left\|H_{1}\right\|_{\mathcal{H}_{\varepsilon}^{k}(\lambda)}, \\
& \left\|H_{1}^{*} H_{3}\right\|_{L\left(\mathbf{C}^{k^{\prime}}, \mathbf{C}^{k}\right)} \leq\left\|H_{1}\right\|_{\mathcal{H}_{\varepsilon}^{k}(\lambda)}\left\|H_{3}\right\|_{\mathcal{H}_{\varepsilon}^{k^{\prime}}(\lambda)} .
\end{aligned}
$$

Also, the following lemma has been proved in Métivier [8, Lemma A.3].

Lemma 3.12. There exists a constant $M_{0}$ such that for all $0<\varepsilon^{\prime}<\varepsilon \leq 1$ and all $j= \pm 1, \ldots, \pm d$,

for all $h \in H_{\varepsilon}(\lambda)$.

$$
\left\|T_{j}(h)\right\|_{H_{\varepsilon^{\prime}}(\lambda)} \leq\left(\frac{M_{0}}{\varepsilon-\varepsilon^{\prime}}\right)^{1 / 2}\|h\|_{H_{\varepsilon}(\lambda)}
$$

Finally, we set $\mathcal{H}_{\varepsilon}^{ \pm}(\lambda)=\mathcal{H}_{\varepsilon}^{k_{ \pm}}(\lambda)$ and $\mathcal{H}_{\varepsilon}^{ \pm *}(\lambda)=\mathcal{H}_{\varepsilon}^{k_{ \pm *}}(\lambda)$.

\section{Construction of parametrix}

4.1. The case $c_{\alpha \beta}=c_{\alpha \beta}\left(y, D_{y}\right)$. Let $P=\sum_{|\alpha|+|\beta| \leq m} t^{\alpha} c_{\alpha \beta}\left(x, D_{x}\right) D_{t}^{\beta}$ be an operator of the form (2.4) satisfying (2.5). Multiplying $P$ by an elliptic factor we may assume $\mu=m / 2$. Also we suppose $m \geq d+1$ in the construction of a parametrix. Otherwise we replace $P$ by $P\left(P^{*} P+1\right)^{k}$ for some integer $k$. Because $\left(P^{*} P+1\right)^{k}$ is isomorphic on $\mathcal{C}_{\mathbf{R}^{N}}^{f}\left(\stackrel{\circledR}{x}^{*}\right)$, this does not affect the conclusion of Theorem 2.1. Moreover, we assume in this section

$$
c_{\alpha \beta}(x, \xi)=c_{\alpha \beta}(y, \eta) \quad \text { independent of } t, \tau .
$$

Then $c_{\alpha \beta}\left(y^{*}\right)=\sum_{j=0}^{\infty} c_{\alpha \beta, j}\left(y^{*}\right) \in S_{\mathrm{phg}}^{(|\alpha|-|\beta|) / 2,1 / 2}\left(\widetilde{\omega}_{\rho}\right)$, where $\widetilde{\omega}_{\rho}$ is a conic complex neigh-

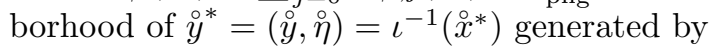

$$
\omega_{\rho}=\left\{(y, \eta) \in \mathbf{C}^{n} \times\left(\mathbf{C}^{n} \backslash 0\right) ;|y-\stackrel{\circ}{y}|<\rho,|\eta-\grave{\eta}|<\rho\right\}
$$

and $c_{\alpha \beta, j}$ is positively homogeneous of degree $(|\alpha|-|\beta|-j) / 2$.

Now, we set

$$
P_{j}\left(y^{*}\right)=\sum_{|\alpha|+|\beta| \leq m} c_{\alpha \beta, j}\left(y^{*}\right) t^{\alpha} D_{t}^{\beta}
$$


Then $P_{j} \in \mathcal{O}^{(-j / 2)}\left(\widetilde{\omega}_{\rho} ; \mathcal{A}^{m}\right)$ and

$$
P\left(y^{*}\right)\left(\stackrel{\text { def }}{=} \sum_{|\alpha|+|\beta| \leq m} c_{\alpha \beta}\left(y^{*}\right) t^{\alpha} D_{t}^{\beta}\right)=\sum_{j=0}^{\infty} P_{j}\left(y^{*}\right) \in S_{\mathrm{phg}}^{0,1 / 2}\left(\widetilde{\omega}_{\rho} ; \mathcal{A}^{m}\right) .
$$

For $y^{*} \in \widetilde{\omega}_{\rho}$, we let $P_{0}^{*}\left(y^{*}\right)=\left(P_{0}\left(\bar{y}^{*}\right)\right)^{*}$ and write $P_{0}^{*} P_{0}\left(y^{*}\right)=P_{0}^{*}\left(y^{*}\right) P_{0}\left(y^{*}\right)$ and $P_{0} P_{0}^{*}\left(y^{*}\right)=P_{0}\left(y^{*}\right) P_{0}^{*}\left(y^{*}\right)$. By the assumption $(2.4), P_{0}^{*} P_{0}\left(\grave{y}^{*}\right)$ and $P_{0} P_{0}^{*}\left(\grave{y}^{*}\right)$ are Fredholm operators from $\mathcal{S}^{\prime}\left(\mathbf{R}^{d}\right)$ to $\mathcal{S}^{\prime}\left(\mathbf{R}^{d}\right)$ together with $P_{0}\left(\ddot{y}^{*}\right)$. (Note that $P_{0}\left(\stackrel{\circ}{ }^{*}\right)=$ $\widehat{\sigma}_{\Sigma}(P)_{\stackrel{\vartheta}{*}^{*}}$.)

Let $\gamma \subset \mathbf{C}$ be a positively oriented closed curve enclosing only the 0-eigenvalue of $P_{0}^{*} P_{0}\left(\stackrel{(}{ }^{*}\right)$ and $P_{0} P_{0}^{*}\left(\stackrel{(}{y}^{*}\right)$. If $\rho>0$ is sufficiently small then for all $y^{*} \in \widetilde{\omega}_{\rho}$ and all $\zeta \in \gamma$, $P_{0}^{*} P_{0}\left(y^{*}\right)-\zeta$ and $P_{0} P_{0}^{*}\left(y^{*}\right)-\zeta$ are invertible. So we set for $y^{*} \in \widetilde{\omega}_{\rho}$,

$$
\begin{aligned}
Q_{0}\left(y^{*}\right) & =\frac{1}{2 \pi i}\left(\int_{\gamma} \zeta^{-1}\left(P_{0}^{*} P_{0}\left(y^{*}\right)-\zeta\right)^{-1} d \zeta\right) P_{0}^{*}\left(y^{*}\right), \\
\Pi_{0}^{+}\left(y^{*}\right) & =\frac{-1}{2 \pi i} \int_{\gamma}\left(P_{0}^{*} P_{0}\left(y^{*}\right)-\zeta\right)^{-1} d \zeta, \\
\Pi_{0}^{-}\left(y^{*}\right) & =\frac{-1}{2 \pi i} \int_{\gamma}\left(P_{0} P_{0}^{*}\left(y^{*}\right)-\zeta\right)^{-1} d \zeta, \\
\mathrm{E}_{0}^{ \pm}\left(y^{*}\right) & =\Pi_{0}^{ \pm}\left(\mathcal{S}^{\prime}\left(\mathbf{R}^{d}\right)\right) .
\end{aligned}
$$

Note that $\Pi_{0}^{+}\left(\stackrel{\circ}{ }^{*}\right)\left(\right.$ resp. $\left.\Pi_{0}^{-}\left(\stackrel{\circ}{y}^{*}\right)\right)$ are the projections onto $\operatorname{Ker}\left(P_{0}\left(\stackrel{\circ}{y}^{*}\right)\right)\left(\operatorname{resp} \operatorname{Ker}\left(P_{0}^{*}\left(\stackrel{\circ}{ }^{*}\right)\right)\right.$ $\left.\simeq \operatorname{Coker}\left(P_{0}\left(\stackrel{\circ}{ }^{*}\right)\right)\right)$. Also, from the choice of $\rho, \operatorname{dim}\left(E_{0}^{ \pm}\left(y^{*}\right)\right)$ is constant for $y^{*} \in \widetilde{\omega}_{\rho}$, hence equal to $k_{ \pm}$.

Then we have

Proposition 4.1 (Métivier [8], Proposition 2.3). There exist $\rho_{0}>0$ and $R_{0}>0$ such that

$$
Q_{0}\left(y^{*}\right) \in \mathcal{O}^{(0)}\left(\widetilde{\omega}_{\rho_{0}} ; \mathcal{L}_{R_{0}}^{m}\right) .
$$

Proposition 4.2. We can choose bases $\left\{h_{0, l}^{+}\left(t ; y^{*}\right)\right\}_{l=1}^{k_{+}}\left(\right.$resp. $\left.\quad\left\{h_{0, l}^{-}\left(t ; y^{*}\right)\right\}_{l=1}^{k_{-}}\right)$of $\mathrm{E}_{0}^{+}\left(y^{*}\right)$ (resp. $\left.\mathrm{E}_{0}^{-}\left(y^{*}\right)\right)$ in $L^{2}\left(\mathbf{R}^{d}\right)$ which are orthonormal if $y^{*}$ is real and such that

$$
h_{0, l}^{ \pm}\left(t ; y^{*}\right) \in \mathcal{O}^{(0)}\left(\widetilde{\omega}_{\rho_{0}} ; H_{\varepsilon_{0}}\right), \quad l=1, \ldots, k_{ \pm},
$$

for some $\rho_{0}>0$ and $\varepsilon_{0}>0$.

Proof. It follows from Theorem 3.9 in Chap. VII of Kato [6] that we can choose bases $\left\{h_{0, l}^{+}\left(t ; y^{*}\right)\right\}_{l=1}^{k_{+}}$(resp. $\left.\left\{h_{0, l}^{-}\left(t ; y^{*}\right)\right\}_{l=1}^{k_{-}}\right)$of $\mathrm{E}_{0}^{+}\left(y^{*}\right)$ (resp. $\left.\mathrm{E}_{0}^{-}\left(y^{*}\right)\right)$, depending holomorphically on $y^{*} \in \widetilde{\omega}_{\rho_{0}}$, orthonormal for real $y^{*}$. Then, for each fixed $y^{*}, h_{0, l}^{ \pm}\left(t ; y^{*}\right)$ are in $\mathcal{H}_{\varepsilon_{0}}$ for some $\varepsilon_{0}>0$. (See e.g. Melin [9, Lemma A.1].)

Let $\left\{h_{0, l}^{ \pm}\left(t ; y^{*}\right)\right\}_{l=1}^{k_{-}}$be chosen as above and define the operators $H_{0}^{ \pm} \in \mathcal{H}_{\varepsilon_{0}}^{ \pm}$and $H_{0}^{ \pm *} \in$ $\mathcal{H}_{\varepsilon_{0}}^{ \pm *}$ by

$$
\begin{aligned}
& H_{0}^{ \pm}: \mathbf{C}^{k} \ni\left(z_{l}\right)_{l=1}^{k_{ \pm}} \mapsto \sum_{l=1}^{k_{ \pm}} z_{l} h_{0, l}^{ \pm}\left(t ; y^{*}\right) \in \mathcal{S}\left(\mathbf{R}^{d}\right), \\
& H_{0}^{ \pm *}: \mathcal{S}^{\prime}\left(\mathbf{R}^{d}\right) \ni u(t) \mapsto\left(\int_{\mathbf{R}^{d}} \overline{h_{0, l}^{ \pm}\left(t, \bar{y}^{*}\right)} u(t) d t\right)_{l=1}^{k_{ \pm}} \in \mathbf{C}^{k_{ \pm}} .
\end{aligned}
$$


Then we have

$$
\Pi_{0}^{ \pm}\left(y^{*}\right)=H_{0}^{ \pm}\left(y^{*}\right) H_{0}^{ \pm *}\left(y^{*}\right) .
$$

Let us also introduce a matrix

$$
M_{0}\left(y^{*}\right)=-H_{0}^{-*}\left(y^{*}\right) P_{0}\left(y^{*}\right) H_{0}^{+}\left(y^{*}\right) .
$$

Then, by Lemma 3.11 and Lemma 3.12,

$$
M_{0}\left(y^{*}\right) \in \mathcal{O}^{(0)}\left(\widetilde{\omega}_{\rho_{0}} ; \mathcal{M}^{ \pm}\right)
$$

and we have

Proposition 4.3. There is a $\rho_{0}>0$ such that for all $y^{*} \in \widetilde{\omega}_{\rho_{0}}$,

$$
\begin{aligned}
& \left(\begin{array}{cc}
P_{0}\left(y^{*}\right) & H_{0}^{-}\left(y^{*}\right) \\
H_{0}^{+*}\left(y^{*}\right) & 0
\end{array}\right)\left(\begin{array}{cc}
Q_{0}\left(y^{*}\right) & H_{0}^{+}\left(y^{*}\right) \\
H_{0}^{-*}\left(y^{*}\right) & M_{0}\left(y^{*}\right)
\end{array}\right)=\left(\begin{array}{cc}
\operatorname{Id}_{\mathcal{S}^{\prime}\left(\mathbf{R}^{d}\right)} & 0 \\
0 & \operatorname{Id}_{\mathbf{C}^{k_{+}}}
\end{array}\right), \\
& \left(\begin{array}{cc}
Q_{0}\left(y^{*}\right) & H_{0}^{+}\left(y^{*}\right) \\
H_{0}^{-*}\left(y^{*}\right) & M_{0}\left(y^{*}\right)
\end{array}\right)\left(\begin{array}{cc}
P_{0}\left(y^{*}\right) & H_{0}^{-}\left(y^{*}\right) \\
H_{0}^{+*}\left(y^{*}\right) & 0
\end{array}\right)=\left(\begin{array}{cc}
\operatorname{Id}_{\mathcal{S}^{\prime}\left(\mathbf{R}^{d}\right)} & 0 \\
0 & \operatorname{Id}_{\mathbf{C}^{k_{-}}}
\end{array}\right) .
\end{aligned}
$$

Proof. This is an easy consequence of the resolvent equation. (See e.g. Kato [6, I- $\$ 5.3]$.

We write

where

$$
L\left(y^{*}\right)=\left(\begin{array}{cc}
P\left(y^{*}\right) & H_{0}^{-}\left(y^{*}\right) \\
H_{0}^{+*}\left(y^{*}\right) & 0
\end{array}\right)=\sum_{j=0}^{\infty} L_{j}\left(y^{*}\right),
$$

$$
L_{0}\left(y^{*}\right)=\left(\begin{array}{cc}
P_{0}\left(y^{*}\right) & H_{0}^{-}\left(y^{*}\right) \\
H_{0}^{+*}\left(y^{*}\right) & 0
\end{array}\right), \quad L_{j}\left(y^{*}\right)=\left(\begin{array}{cc}
P_{j}\left(y^{*}\right) & 0 \\
0 & 0
\end{array}\right) \quad \text { for } j \geq 1
$$

and construct a right parametrix $E\left(y^{*}\right)=\sum_{j=0}^{\infty} E_{j}\left(y^{*}\right)$ of $L\left(y^{*}\right)$ so that

$$
L \# E=\sum_{l=0}^{\infty} \sum_{i+j+2|\alpha|=l} \frac{1}{\alpha !}\left(\partial_{\eta}^{\alpha} L_{i}\right)\left(D_{y}^{\alpha} E_{j}\right)=\left(\begin{array}{ll}
\mathrm{I} & 0 \\
0 & \mathrm{I}
\end{array}\right) \text {, }
$$

where \# denotes the pseudo-differential composition of symbols in $(y, \eta)$.

By Proposition 4.3 we can take

$$
E_{0}\left(y^{*}\right)=\left(\begin{array}{cc}
Q_{0}\left(y^{*}\right) & H_{0}^{+}\left(y^{*}\right) \\
H_{0}^{-*}\left(y^{*}\right) & M_{0}\left(y^{*}\right)
\end{array}\right) .
$$

Then, for $j \geq 1, E_{l}$ 's are determined recurrently by

$$
E_{l}\left(y^{*}\right)=-\sum_{\substack{i+j+2|\alpha|=l \\ j \leq l-1}} \frac{1}{\alpha !} E_{0}\left(y^{*}\right)\left(\partial_{\eta}^{\alpha} L_{i}\left(y^{*}\right)\right)\left(D_{y}^{\alpha} E_{j}\left(y^{*}\right)\right) .
$$

We want to show $\sum_{j=0}^{\infty} E_{j}$ has a meaning as a formal sum of operator valued analytic pseudo-differential operators. For this purpose we introduce a norm for $E_{j}$ as follows:

Definition 4.4. For $\varepsilon>0$ and $\rho>0, \mathcal{E}_{\varepsilon, \rho}^{(\mu)}$ denotes the space of operator valued symbols on $\widetilde{\omega}_{\rho}$ of the form

$$
E\left(y^{*}\right)=\left(\begin{array}{cc}
Q\left(y^{*}\right) & H^{+}\left(y^{*}\right) \\
H^{-*}\left(y^{*}\right) & M\left(y^{*}\right)
\end{array}\right) \in\left(\begin{array}{cc}
\mathcal{O}^{(\mu)}\left(\widetilde{\omega}_{\rho} ; \mathcal{B}_{\varepsilon}\right) & \mathcal{O}^{(\mu)}\left(\widetilde{\omega}_{\rho} ; \mathcal{H}_{\varepsilon}^{+}\right) \\
\mathcal{O}^{(\mu)}\left(\widetilde{\omega}_{\rho} ; \mathcal{H}_{\varepsilon}^{-*}\right) & \mathcal{O}^{(\mu)}\left(\widetilde{\omega}_{\rho} ; \mathcal{M}^{ \pm}\right)
\end{array}\right)
$$


The norm of $\mathcal{E}_{\varepsilon, \rho}^{(\mu)}$ is defined by

$$
\|E\|_{\mathcal{E}_{\varepsilon, \rho}^{(\mu)}}=\max \left\{\|Q\|_{\mathcal{B}_{\varepsilon, \rho}^{(\mu)}},\left\|H^{+}\right\|_{\mathcal{H}_{\varepsilon, \rho}^{+,(\mu)}},\left\|H^{-*}\right\|_{\mathcal{H}_{\varepsilon, \rho}^{-*,(\mu)}},\|M\|_{\mathcal{M}_{\rho}^{ \pm,(\mu)}}\right\} .
$$

We have

Lemma 4.5. Suppose $m \geq d+1$. Then there exist $\varepsilon_{0}, \rho_{0}$ and $C$ such that for all $0<\rho<\rho_{0}$,

$$
\left\|E_{j}\right\|_{\mathcal{E}_{\varepsilon_{0}, \rho}^{(-j / 2)}} \leq C\left(\frac{C j}{\rho_{0}-\rho}\right)^{j / 2}
$$

for $j=0,1,2, \ldots$

Proof. By Proposition 4.1, $Q_{0}$ is in $\mathcal{O}^{(0)}\left(\widetilde{\omega}_{\rho_{0}} ; \mathcal{L}_{R_{0}}^{m}\right)$ for some $\rho_{0}>0, R_{0}>0$. Then by Lemma 3.6 there is a $\varepsilon_{0}$ for which we have $Q_{0} \in \mathcal{O}^{(0)}\left(\widetilde{\omega}_{\rho_{0}} ; \mathcal{B}_{\varepsilon_{0}}\right)$. Hence, together with Proposition 4.2 and (4.2), $E_{0}$ is in $\mathcal{E}_{\varepsilon_{0}, \rho_{0}}^{(0)}$ by decreasing $\varepsilon_{0}$ if necessary. Here, for later convenience, we suppose $\varepsilon_{0}$ is so chosen that Lemma 3.7 holds. Also we can assume the following estimates are satisfied for a constant $C_{0}$ :

$$
\begin{gathered}
\left\|\partial_{\eta}^{\alpha} P_{i}\right\|_{\mathcal{A}_{\rho_{0}}^{m,(-|\alpha|-i / 2)}} \leq C_{0}^{|\alpha|+i / 2+1} \alpha !(i !)^{1 / 2}, \\
\left\|\partial_{\eta}^{\alpha} H_{0}^{ \pm}\right\|_{\mathcal{H}_{\varepsilon_{0}, \rho_{0}}^{ \pm,(-\alpha \mid)}} \leq C_{0}^{|\alpha|+1} \alpha ! \\
\left\|Q_{0}\right\|_{\mathcal{L}_{R_{0}, \rho_{0}}^{m,(0)}} \leq C_{0}, \quad\left\|Q_{0}\right\|_{\mathcal{B}_{\varepsilon_{0}, \rho_{0}}^{(0)}} \leq C_{0}, \\
\left\|M_{0}\right\|_{\mathcal{M}_{\rho_{0}}^{ \pm,(0)}} \leq C_{0} .
\end{gathered}
$$

For $j \geq 1$, we shall prove (4.5) by induction. First we note that if $E_{j} \in \mathcal{E}_{\varepsilon_{0}, \rho}^{(-j / 2)}$ then, by Cauchy's inequality, there is an $M_{0}$ which depends only on $d$ such that for all $0<\rho^{\prime}<\rho<\rho_{0}$,

$$
\left\|D_{y}^{\alpha} E_{j}\right\|_{\mathcal{E}_{\varepsilon_{0}, \rho^{\prime}}^{(-j / 2)}} \leq\left(\frac{M_{0}|\alpha|}{\rho-\rho^{\prime}}\right)^{|\alpha|}\left\|E_{j}\right\|_{\mathcal{E}_{\varepsilon_{0}, \rho}^{(-j / 2)}} .
$$

We write (4.4) as

where

$$
E_{l}=-\sum_{k=1}^{l} \mathcal{M}_{k}\left(E_{l-k}\right)
$$

Then we have

$$
\mathcal{M}_{k}\left(E_{j}\right)=\sum_{2|\alpha|+i=k} \frac{1}{\alpha !} E_{0}\left(\partial_{\eta}^{\alpha} L_{i}\right)\left(D_{y}^{\alpha} E_{j}\right)
$$

$$
\begin{aligned}
\mathcal{M}_{k}^{11}\left(E_{j}\right)= & \sum_{2|\alpha|+i=k} \frac{1}{\alpha !} Q_{0} \partial_{\eta}^{\alpha} P_{i} D_{y}^{\alpha} Q_{j} \\
& +\sum_{2|\alpha|=k} \frac{1}{\alpha !}\left(H_{0}^{+} \partial_{\eta}^{\alpha} H_{0}^{+*} D_{y}^{\alpha} Q_{j}+Q_{0} \partial_{\eta}^{\alpha} H_{0}^{-} D_{y}^{\alpha} H_{j}^{-*}\right), \\
\mathcal{M}_{k}^{12}\left(E_{j}\right)= & \sum_{2|\alpha|+i=k} \frac{1}{\alpha !} Q_{0} \partial_{\eta}^{\alpha} P_{i} D_{y}^{\alpha} H_{j}^{+} \\
& +\sum_{2|\alpha|=k} \frac{1}{\alpha !}\left(H_{0}^{+} \partial_{\eta}^{\alpha} H_{0}^{+*} D_{y}^{\alpha} H_{j}^{+}+Q_{0} \partial_{\eta}^{\alpha} H_{0}^{-} D_{y}^{\alpha} M_{j}\right),
\end{aligned}
$$




$$
\begin{aligned}
\mathcal{M}_{k}^{21}\left(E_{j}\right)= & \sum_{2|\alpha|+i=k} \frac{1}{\alpha !} H_{0}^{-*} \partial_{\eta}^{\alpha} P_{i} D_{y}^{\alpha} Q_{j} \\
& +\sum_{2|\alpha|=k} \frac{1}{\alpha !}\left(M_{0} \partial_{\eta}^{\alpha} H_{0}^{+*} D_{y}^{\alpha} Q_{j}+H_{0}^{-*} \partial_{\eta}^{\alpha} H_{0}^{-} D_{y}^{\alpha} H_{j}^{-*}\right), \\
\mathcal{M}_{k}^{22}\left(E_{j}\right)= & \sum_{2|\alpha|+i=k} \frac{1}{\alpha !} H_{0}^{-*} \partial_{\eta}^{\alpha} P_{i} D_{y}^{\alpha} H_{j}^{+} \\
& +\sum_{2|\alpha|=k} \frac{1}{\alpha !}\left(M_{0} \partial_{\eta}^{\alpha} H_{0}^{+*} D_{y} H_{j}^{+}+H_{0}^{-*} \partial_{\eta}^{\alpha} H_{0}^{-} D_{y}^{\alpha} M_{j}\right) .
\end{aligned}
$$

We shall show that there exists an $M$ such that for all $0<\rho^{\prime}<\rho<\rho_{0}$,

$$
\left\|\mathcal{M}_{k}\left(E_{j}\right)\right\|_{\mathcal{E}_{\varepsilon_{0}, \rho^{\prime}}^{(-j / 2-k / 2)}} \leq M\left(\frac{M k}{\rho-\rho^{\prime}}\right)^{k / 2}\left\|E_{j}\right\|_{\mathcal{E}_{\varepsilon_{0}, \rho}^{(-j / 2)}} .
$$

By Lemmas 3.7 and $3.11, \mathcal{M}_{k}^{11}\left(E_{j}\right)$ is in $\mathcal{O}^{(-j / 2-k / 2)}\left(\widetilde{\omega}_{\rho^{\prime}} ; \mathcal{B}_{\varepsilon_{0}}\right)$ and we have

$$
\begin{aligned}
& \left\|\mathcal{M}_{k}^{11}\left(E_{j}\right)\right\|_{\mathcal{B}_{\varepsilon_{0}, \rho^{\prime}}^{(-j / 2-k / 2)}} \\
& \leq \sum_{2|\alpha|+i=k} \frac{C_{1} C_{2}}{\alpha !}\left\|Q_{0}\right\|_{\mathcal{L}_{R_{0}, \rho_{0}}^{m,(0)}}\left\|\partial_{\eta}^{\alpha} P_{i}\right\|_{\mathcal{A}_{\rho_{0}}^{m,(-|\alpha|-i / 2)}}\left\|D_{y}^{\alpha} Q_{j}\right\|_{\mathcal{B}_{\varepsilon_{0}, \rho^{\prime}}^{(-j / 2)}} \\
& +\sum_{2|\alpha|=k} \frac{1}{\alpha !}\left(\left\|H_{0}^{+}\right\|_{\mathcal{H}_{\varepsilon_{0}, \rho_{0}}^{+,(0)}}\left\|\partial_{\eta}^{\alpha} H_{0}^{+*}\right\|_{\mathcal{H}_{\varepsilon_{0}, \rho_{0}}^{+*,(-|\alpha|)}}\left\|D_{y}^{\alpha} Q_{j}\right\|_{\mathcal{B}_{\varepsilon_{0}, \rho^{\prime}}^{(-j / 2)}}\right. \\
& \left.+\left\|Q_{0}\right\|_{\mathcal{B}_{\varepsilon_{0}, \rho_{0}}^{(0)}}\left\|\partial_{\eta}^{\alpha} H_{0}^{-}\right\|_{\mathcal{H}_{\varepsilon_{0}, \rho_{0}}^{-,(-|\alpha|)}}\left\|D_{y}^{\alpha} H_{j}^{-*}\right\|_{\mathcal{H}_{\varepsilon_{0}, \rho^{\prime}}^{-*,-1 / 2)}}\right) \\
& \leq \sum_{2|\alpha|+i=k} C_{1} C_{2} C_{0}^{2} C_{0}^{|\alpha|+i / 2}(i !)^{1 / 2}\left(\frac{M_{0}|\alpha|}{\rho-\rho^{\prime}}\right)^{|\alpha|}\left\|Q_{j}\right\|_{\mathcal{B}_{\varepsilon_{0}, \rho}^{(-j / 2)}} \\
& +\sum_{2|\alpha|=k} C_{0}^{2} C_{0}^{|\alpha|}\left(\frac{M_{0}|\alpha|}{\rho-\rho^{\prime}}\right)^{|\alpha|}\left\|Q_{j}\right\|_{\mathcal{B}_{\varepsilon_{0}, \rho}^{(-j / 2)}} \\
& +\sum_{2|\alpha|=k} C_{0}^{2} C_{0}^{|\alpha|}\left(\frac{M_{0}|\alpha|}{\rho-\rho^{\prime}}\right)^{|\alpha|}\left\|H_{j}^{-*}\right\|_{\mathcal{H}_{\varepsilon_{0}, \rho}^{-*,(-j / 2)}} \\
& \leq\left(C_{1} C_{2} C_{0}^{2}\left(\frac{C_{0} M_{0}(n+1) k}{\rho-\rho^{\prime}}\right)^{k / 2}+2 C_{0}^{2}\left(\frac{C_{0} M_{0} n k}{\rho-\rho^{\prime}}\right)^{k / 2}\right)\left\|E_{j}\right\|_{\mathcal{E}_{\varepsilon_{0}, \rho}^{(-j / 2)}} \\
& \leq M\left(\frac{M k}{\rho-\rho^{\prime}}\right)^{k / 2}\left\|E_{j}\right\|_{\mathcal{E}_{\varepsilon_{0}, \rho}^{(-j / 2)}},
\end{aligned}
$$

provided $M \geq \max \left\{\left(C_{1} C_{2}+2\right) C_{0}^{2}, C_{0} M_{0}(n+1)\right\}$, where $C_{1}$ is a constant appearing in (3.1) and $C_{2}$ is a constant appearing in Lemma 3.7.

$\mathcal{M}_{k}^{12}\left(E_{j}\right)$ can be estimated in the same way by using Lemma 3.12 instead of Lemma 3.7 .

To estimate $\mathcal{M}_{k}^{21}\left(E_{j}\right)$ we suppose further that

$$
\left\|H_{0}^{-*}\right\|_{\mathcal{H}_{2 \varepsilon_{0}, \rho_{0}}^{-*,(0)}} \leq C_{0}
$$


(We need only replace $\varepsilon_{0}$ by $\varepsilon_{0} / 2$.) Then by Lemma 3.13 we have, for $A \in \mathcal{A}_{\rho_{0}}^{m,(\mu)}$,

$$
\left\|H_{0}^{-*} A\right\|_{\mathcal{H}_{\varepsilon_{0}, \rho_{0}}^{-*,(\mu)}} \leq\left(\frac{M_{0} m}{\varepsilon_{0}}\right)^{m / 2}\left\|H_{0}^{-*}\right\|_{\mathcal{H}_{2 \varepsilon_{0}}^{(0)}}\|A\|_{\mathcal{A}_{\rho}^{m,(\mu)}} \leq C_{3} C_{0}\|A\|_{\mathcal{A}_{\rho}^{m,(\mu)}} .
$$

Here we set $C_{3}=\left(M_{0} m / \varepsilon_{0}\right)^{m / 2}$. We have

$$
\begin{aligned}
& \left\|\mathcal{M}_{k}^{21}\left(E_{j}\right)\right\|_{\mathcal{H}_{\varepsilon_{0}, \rho^{\prime}}^{*, j / 2-k / 2)}} \\
& \leq \sum_{2|\alpha|+i=k} \frac{1}{\alpha !}\left\|H_{0}^{-*} \partial_{\eta}^{\alpha} P_{i}\right\|_{\mathcal{H}_{\varepsilon_{0}, \rho_{0}}^{-*,(-|\alpha|-i / 2)}}\left\|D_{y}^{\alpha} Q_{j}\right\|_{\mathcal{B}_{\varepsilon_{0}, \rho^{\prime}}^{(-j / 2)}} \\
& +\sum_{2|\alpha|=k} \frac{1}{\alpha !}\left(\left\|M_{0}\right\|_{\mathcal{M}_{\varepsilon_{0}, \rho_{0}}^{ \pm,(0)}}\left\|\partial_{\eta}^{\alpha} H_{0}^{+*}\right\|_{\mathcal{H}_{\varepsilon_{0}, \rho_{0}}^{+*,(-|\alpha|)}}\left\|D_{y}^{\alpha} Q_{j}\right\|_{\mathcal{B}_{\varepsilon_{0}, \rho^{\prime}}^{(-j / 2)}}\right. \\
& \left.+\left\|H_{0}^{-*}\right\|_{\mathcal{H}_{\varepsilon_{0}, \rho_{0}}^{-*,(j)}}\left\|\partial_{\eta}^{\alpha} H_{0}^{-}\right\|_{\mathcal{H}_{\varepsilon_{0}, \rho_{0}}^{-,(-|\alpha|)}}\left\|D_{y}^{\alpha} H_{j}^{-*}\right\|_{\mathcal{H}_{\varepsilon_{0}, \rho^{\prime}}^{-*,(-j / 2)}}\right) \\
& \leq \sum_{2|\alpha|+i=k} C_{3} C_{0}^{2} C_{0}^{|\alpha|+i / 2}(i !)^{1 / 2}\left(\frac{M_{0}|\alpha|}{\rho-\rho^{\prime}}\right)^{|\alpha|}\left\|Q_{j}\right\|_{\mathcal{B}_{\varepsilon_{0}, \rho}^{(-j / 2)}} \\
& +\sum_{2|\alpha|=k} C_{0}^{2} C_{0}^{|\alpha|}\left(\frac{M_{0}|\alpha|}{\rho-\rho^{\prime}}\right)^{|\alpha|}\left\|Q_{j}\right\|_{\mathcal{B}_{\varepsilon_{0}, \rho}^{(-j / 2)}} \\
& +\sum_{2|\alpha|=k} C_{0}^{2} C_{0}^{|\alpha|}\left(\frac{M_{0}|\alpha|}{\rho-\rho^{\prime}}\right)^{|\alpha|}\left\|H_{j}^{-*}\right\|_{\mathcal{H}_{\varepsilon_{0}, \rho}^{-*,(-j / 2)}} \\
& \leq\left(C_{3} C_{0}^{2}\left(\frac{C_{0} M_{0}(n+1) k}{\rho-\rho^{\prime}}\right)^{k / 2}+2 C_{0}^{2}\left(\frac{C_{0} M_{0} n k}{\rho-\rho^{\prime}}\right)^{k / 2}\right)\left\|E_{j}\right\|_{\mathcal{E}_{\varepsilon_{0} \cdot \rho}^{(-j / 2)}} \\
& \leq M\left(\frac{M k}{\rho-\rho^{\prime}}\right)^{k / 2}\left\|E_{j}\right\|_{\mathcal{E}_{\varepsilon_{0}, \rho}^{(-j / 2)}},
\end{aligned}
$$

provided $M \geq \max \left\{\left(C_{3}+2\right) C_{0}^{2}, C_{0} M_{0}(n+1)\right\}$.

$\mathcal{M}_{k}^{22}\left(E_{j}\right)$ can be estimated in the same way and we have proved (4.11).

Now assume that (4.5) has been proved up to order $j=l-1$. Using (4.11) with $\rho=\rho^{\prime}+(k / l)\left(\rho_{0}-\rho^{\prime}\right)$ we obtain

$$
\begin{aligned}
\left\|\mathcal{M}_{k}\left(E_{l-k}\right)\right\|_{\mathcal{E}_{\varepsilon_{0}, \rho^{\prime}}^{(-l / 2)}} & \leq M\left(\frac{M k}{\rho-\rho^{\prime}}\right)^{k / 2}\left\|E_{l-k}\right\|_{\mathcal{E}_{\varepsilon_{0}, \rho}^{(-l / 2+k / 2)}} \\
& \leq M\left(\frac{M k}{\rho-\rho^{\prime}}\right)^{k / 2} C\left(\frac{C(l-k)}{\rho_{0}-\rho}\right)^{(l-k) / 2} \\
& \leq C\left(\frac{C l}{\rho_{0}-\rho^{\prime}}\right)^{l / 2} M\left(\frac{M}{C}\right)^{k / 2}
\end{aligned}
$$

Therefore, $E_{l}=-\sum_{k=1}^{l} \mathcal{M}_{k}\left(E_{l-k}\right)$ satisfies

$$
\left\|E_{l}\right\|_{\mathcal{E}_{\varepsilon_{0}, \rho^{\prime}}^{(-l / 2)}} \leq C\left(\frac{C l}{\rho_{0}-\rho^{\prime}}\right)^{l / 2} M \sum_{k=1}^{l}\left(\frac{M}{C}\right)^{k / 2},
$$

which implies (4.5) at order $j=l$, if $C$ is large enough $\left(C \geq \max \left\{4 M, 4 M^{3}\right\}\right)$. 
In the same way we can construct a left parametrix of $L$ and find that the above $E$ is a two-side parametrix of $L$.

4.2. General case. In this section we remove the assumption (4.1) and describe needed modifications in the construction of a relative parametrix.

Let

$$
P=\sum_{|\alpha|+|\beta| \leq m} t^{\alpha} c_{\alpha \beta}\left(x, D_{x}\right) D_{t}^{\beta}
$$

be an operator of order $\mu=m / 2$ of the form (2.4) satisfying $(2.5)$, where $c_{\alpha \beta}(x, \xi)=$ $\sum_{j=0}^{\infty} c_{\alpha \beta, j}(x, \xi)$ is in $a-S_{\mathrm{phg}}^{(|\alpha|-|\beta|) / 2,1 / 2}$ in a conic neighborhood of $\stackrel{\circ}{x}^{*}=(0, \stackrel{\circ}{y}, 0, \stackrel{\imath}{\eta})$. As in Section 4.1 we assume $m \geq d+1$ from the beginning.

After taking Taylor expansion of $c_{\alpha \beta, j}$ in $(t, \tau)$ we set

$$
P_{j}\left(y^{*}\right)=\sum_{i+|\gamma|=j} \sum_{|\alpha|+|\beta| \leq m} \partial_{t}^{\gamma_{-}} \partial_{\tau}^{\gamma_{+}} c_{\alpha \beta, i}(0, y, 0, \eta) t^{\alpha+\gamma_{-}} D_{t}^{\beta+\gamma_{+}} .
$$

Interchanging the order of $t^{\gamma-}$ and $D_{t}$ 's we can write $P_{j}$ in the form

$$
P_{j}\left(y^{*}\right)=\sum_{|\gamma| \leq j} P_{j, \gamma}\left(y^{*}\right) D_{t}^{\gamma_{+}} t^{\gamma_{-}}
$$

with

Then $P_{j, \gamma}$ satisfies

$$
P_{j, \gamma}\left(y^{*}\right) \in \mathcal{O}^{\left(-j / 2-\left|\gamma_{+}\right| / 2+\left|\gamma_{-}\right| / 2\right)}\left(\widetilde{\omega}_{\rho_{0}} ; \mathcal{A}^{m}\right) .
$$

for all $j$ and $\gamma=\left(\gamma_{+}, \gamma_{-}\right)$.

Proceeding just as in Section 4.1, we arrive at the construction of a parametrix $E=$ $\sum_{j=0}^{\infty} E_{j}$ of

$$
L=\sum_{j=0}^{\infty} L_{j}=\left(\begin{array}{cc}
P_{0} & H_{0}^{-} \\
H_{0}^{+*} & 0
\end{array}\right)+\sum_{j=1}^{\infty}\left(\begin{array}{cc}
P_{j} & 0 \\
0 & 0
\end{array}\right)
$$

so that (4.3) is satisfied. Then $E_{j}$ 's must be given by (4.4). It only remains to prove the estimate like Lemma 4.5 so that we can realize $\sum_{j=0}^{\infty} E_{j}$ as an analytic micro-local operator. For this purpose we define $\mathcal{E}_{\rho}^{(\mu)}$ as follows: For $\rho>0$ we write in this section $\mathcal{B}_{\rho}^{(\mu)}=\mathcal{O}^{(\mu)}\left(\widetilde{\omega}_{\rho} ; \mathcal{B}_{\rho}\right), \mathcal{H}_{\rho}^{ \pm,(\mu)}=\mathcal{O}^{(\mu)}\left(\widetilde{\omega}_{\rho} ; \mathcal{H}_{\rho}^{ \pm}\right)$and $\mathcal{H}_{\rho}^{ \pm *,(\mu)}=\mathcal{O}^{(\mu)}\left(\widetilde{\omega}_{\rho} ; \mathcal{H}_{\rho}^{ \pm *}\right)$. We let $\mathcal{B}_{\rho}^{(\mu)} \otimes \mathcal{A}^{l}$ (resp. $\mathcal{H}_{\rho}^{-*,(\mu)} \otimes \mathcal{A}^{l}$ ) denote the space of operator valued symbols for which we can write

$$
Q\left(y^{*}\right)=\sum_{|\gamma| \leq l} Q_{\gamma}\left(y^{*}\right) D_{t}^{\gamma_{+}} t^{\gamma_{-}} \quad\left(\text { resp. } H\left(y^{*}\right)=\sum_{|\gamma| \leq l} H_{\gamma}\left(y^{*}\right) D_{t}^{\gamma_{+}} t^{\gamma_{-}}\right)
$$

with $Q_{\gamma} \in \mathcal{B}_{\rho}^{\left(\mu-\left|\gamma_{+}\right| / 2+\left|\gamma_{-}\right| / 2\right)}$ (resp. $H_{\gamma} \in \mathcal{H}_{\rho}^{-*,\left(\mu-\left|\gamma_{+}\right| / 2+\left|\gamma_{-}\right| / 2\right)}$ ).

Definition 4.6. For $\mu \leq 0$ and $\rho>0, \mathcal{E}_{\rho}^{(\mu)}$ denotes the space of operator valued symbol of the form

$$
E=\left(\begin{array}{cc}
Q & H^{+} \\
H^{-*} & M
\end{array}\right) \in\left(\begin{array}{cc}
\mathcal{B}_{\rho}^{(\mu)} \otimes \mathcal{A}^{2|\mu|} & \mathcal{H}_{\rho}^{+,(\mu)} \\
\mathcal{H}_{\rho}^{-*,(\mu)} \otimes \mathcal{A}^{2|\mu|} & \mathcal{M}_{\rho}^{ \pm,(\mu)}
\end{array}\right) .
$$


Then we can prove the following lemma for the estimate of $E_{j}$ 's.

LEMmA 4.7. There exist $\rho_{0}>0$ and $C>0$ such that for all $0<\rho<\rho_{0}$,

and such that

$$
E_{j}=\left(\begin{array}{cc}
Q_{j} & H_{j}^{+} \\
H_{j}^{-*} & M_{j}
\end{array}\right) \in \mathcal{E}_{\rho}^{(-j / 2)},
$$

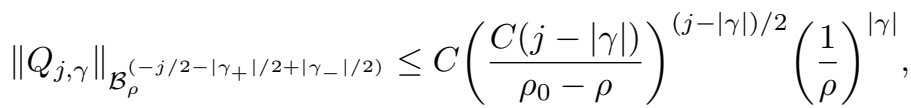

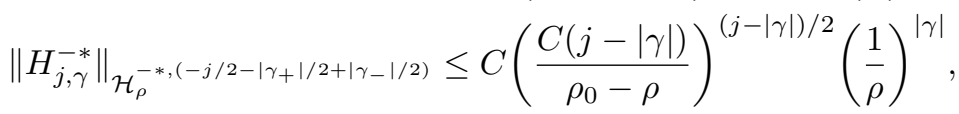

$$
\begin{aligned}
& \left\|H_{j}^{+}\right\|_{\mathcal{H}_{\rho}^{+,(-j / 2)}} \leq C\left(\frac{C j}{\rho_{0}-\rho}\right)^{j / 2}, \\
& \left\|M_{j}\right\|_{\mathcal{M}_{\rho}^{ \pm,(-j / 2)}} \leq C\left(\frac{C j}{\rho_{0}-\rho}\right)^{j / 2},
\end{aligned}
$$

where $Q_{j}=\sum_{|\gamma| \leq j} Q_{j, \gamma} D_{t}^{\gamma_{+}} t^{\gamma_{-}}$and $H_{j}^{-*}=\sum_{|\gamma| \leq j} H_{j, \gamma}^{-*} D_{t}^{\gamma_{+}} t^{\gamma_{-}}$.

The proof of this lemma is straightforward but very long and tedious. So we only describe here how the induction works for $Q_{j}$.

First we note that there is a constant $M_{0}$ such that for all $0<\rho^{\prime}<\rho<1 / 2$ we have

$$
\begin{aligned}
\left\|\left(\operatorname{ad} D_{t}\right)^{\beta_{+}}(\operatorname{ad} t)^{\beta_{-}} D_{y}^{\alpha} Q\right\|_{\mathcal{B}_{\rho^{\prime}}^{\left(\mu-\left|\beta_{+}\right| / 2+\left|\beta_{-}\right| / 2\right)}} & \leq\left(\frac{M_{0}(2|\alpha|+|\beta|)}{\rho-\rho^{\prime}}\right)^{(2|\alpha|+|\beta|) / 2}\|Q\|_{\mathcal{B}_{\rho}^{(\mu)},},\left\|\left(\frac{M_{0}(2|\alpha|+|\beta|)}{\rho-\rho^{\prime}}\right)^{(2|\alpha|+|\beta|) / 2}\right\| H \|_{\mathcal{H}_{\rho}^{ \pm},(\mu)} . \\
\left\|D_{t}^{\beta_{+}} t^{\beta_{-}} D_{y}^{\alpha} H^{ \pm}\right\|_{\mathcal{H}_{\rho^{\prime}}^{ \pm,\left(\mu-\left|\beta_{+}\right| / 2+\left|\beta_{-}\right| / 2\right)}} & \leq(.7)
\end{aligned}
$$

This follows from Lemma 3.8, 3.12 and Cauchy's inequality. We also assume (4.7) through (4.9) in Section 4.1 and (4.13) are satisfied for a constant $C_{0} \geq 1$.

We write

$$
Q_{l}=Q_{l}^{\mathrm{I}}+Q_{l}^{\mathrm{II}}+Q_{l}^{\mathrm{III}}
$$

where

$$
\begin{aligned}
Q_{l}^{\mathrm{I}} & =\sum_{k=1}^{l} \sum_{2|\alpha|+i=k} \frac{1}{\alpha !} Q_{0} \partial_{\eta}^{\alpha} P_{i} D_{y}^{\alpha} Q_{l-k}, \\
Q_{l}^{\mathrm{II}} & =\sum_{k=1}^{l} \sum_{2|\alpha|=k} \frac{1}{\alpha !} H_{0}^{+} \partial_{\eta}^{\alpha} H_{0}^{+*} D_{y}^{\alpha} Q_{l-k}, \\
Q_{l}^{\mathrm{III}} & =\sum_{k=1}^{l} \sum_{2|\alpha|=k} \frac{1}{\alpha !} Q_{0} \partial_{\eta}^{\alpha} H_{0}^{-} D_{y}^{\alpha} H_{l-k}^{-*} .
\end{aligned}
$$

For $Q_{l}^{\mathrm{I}}$, we have

$$
Q_{l}^{\mathrm{I}}=\sum_{k=1}^{l} \sum_{2|\alpha|+i=k} \frac{1}{\alpha !} Q_{0}\left(\sum_{|\beta| \leq i} \partial_{\eta}^{\alpha} P_{i, \beta} D_{t}^{\beta_{+}} t^{\beta_{-}}\right)\left(\sum_{|\gamma| \leq l-k} D_{y}^{\alpha} Q_{l-k, \gamma} D_{t}^{\gamma_{+}} t^{\gamma_{-}}\right) .
$$


Interchanging the order of $D_{t}^{\beta_{+}} t^{\beta_{-}}$and $D_{y}^{\alpha} Q_{l-k, \gamma} D_{t}^{\gamma_{+}}$, we know that the coefficient of $D_{t}^{\gamma_{+}^{\prime}} t^{\gamma_{-}^{\prime}}$ consists of at most $\{3 \sqrt{n+1}(2 d+1)\}^{k}$ terms of the form

$$
\sum_{k=1}^{l} \frac{1}{\alpha !} Q_{0} \partial_{\eta}^{\alpha} P_{i, \beta}\left(\left(\operatorname{ad} D_{t}\right)^{\beta_{1}^{+}}(\operatorname{ad} t)^{\beta_{1}^{-}} D_{y}^{\alpha} Q_{l-k, \gamma}\right) \frac{\gamma_{+} !}{\left(\gamma_{+}-\beta_{2}^{-}\right) !},
$$

where $2|\alpha|+i=k,|\beta| \leq i, \beta_{1}^{+}+\beta_{2}^{+}=\beta_{+}, \beta_{1}^{-}+\beta_{2}^{-}+\beta_{3}^{-}=\beta_{-}, \gamma_{+}=\gamma_{+}^{\prime}-\beta_{2}^{+}+\beta_{2}^{-}$and $\gamma_{-}=\gamma_{-}^{\prime}-\beta_{3}^{-}$.

Now assume (4.14) through (4.17) have been proved up to order $j=l-1$. Then the $\mathcal{B}_{\rho^{\prime}}^{\left(\left(-l-\left|\gamma_{+}^{\prime}\right|+\left|\gamma_{-}^{\prime}\right|\right) / 2\right)}$-norm of each term in (4.18) can be estimated by, for a $\rho \in\left[\rho^{\prime}, \rho_{0}\right)$,

$$
\begin{aligned}
& \frac{1}{\alpha !}\left\|Q_{0}\right\|_{\mathcal{B}_{\rho^{\prime}}^{(0)}}\left\|\partial_{\eta}^{\alpha} P_{i, \beta}\right\|_{\mathcal{A}_{\rho^{\prime}}^{\left(\left(-i-\left|\beta_{+}\right|+\left|\beta_{-}\right|-2|\alpha|\right) / 2\right)}} \\
& \times\left\|\left(\operatorname{ad} D_{t}\right)^{\beta_{1}^{+}}(\operatorname{ad} t)^{\beta_{1}^{-}} D_{y}^{\alpha} Q_{l-k, \gamma}\right\|_{\mathcal{B}_{\rho^{\prime}}^{\left(\left(-l+k-\left|\gamma_{+}\right|+\left|\gamma_{-}\right|+\left|\beta_{1}^{+}\right|-\left|\beta_{1}^{-}\right|\right) / 2\right)}}\left(\frac{\gamma_{+} !}{\left(\gamma_{+}-\beta_{2}^{-}\right) !}\right) \\
& \leq C_{0}^{2} C_{0}^{|\alpha|+\frac{i}{2}} \sqrt{(i-|\beta|) !}\left(\frac{M_{0}\left(2|\alpha|+\left|\beta_{1}^{+}\right|+\left|\beta_{1}^{-}\right|\right)}{\rho-\rho^{\prime}}\right)^{\left(2|\alpha|+\left|\beta_{1}^{+}\right|+\left|\beta_{1}^{-}\right|\right) / 2} \\
& \times C\left(\frac{C(l-k-|\gamma|)}{\rho_{0}-\rho}\right)^{(l-k-|\gamma|) / 2}\left(\frac{1}{\rho}\right)^{|\gamma|} \frac{\gamma_{+} !}{\left(\gamma_{+}-\beta_{2}^{-}\right) !} \\
& \leq C_{0}^{2} C_{0}^{k / 2}\left(\frac{M_{0}\left(k-\left|\beta_{2}^{+}\right|-\left|\beta_{2}^{-}\right|-\left|\beta_{3}^{-}\right|\right)}{\rho-\rho^{\prime}}\right)^{\left(k-\left|\beta_{2}^{+}\right|-\left|\beta_{2}^{-}\right|-\left|\beta_{3}^{-}\right|\right) / 2} \\
& \times C\left(\frac{C\left(l-k-\left|\gamma^{\prime}\right|+\left|\beta_{2}^{+}\right|-\left|\beta_{2}^{-}\right|+\left|\beta_{3}^{-}\right|\right)}{\rho_{0}-\rho}\right)^{\left(l-k-\left|\gamma^{\prime}\right|+\left|\beta_{2}^{+}\right|-\left|\beta_{2}^{-}\right|+\left|\beta_{3}^{-}\right|\right) / 2} \\
& \times\left(\frac{|\beta|_{2}^{-}}{\rho-\rho^{\prime}}\right)^{\left|\beta_{2}^{-}\right|}\left(\frac{1}{\rho^{\prime}}\right)^{\left|\gamma^{\prime}\right|-\left|\beta_{2}^{+}\right|-\left|\beta_{3}^{-}\right|} \\
& \leq C_{0}^{2} C_{0}^{k / 2}\left(\frac{M_{0}\left(k-\left|\beta_{2}^{+}\right|+\left|\beta_{2}^{-}\right|-\left|\beta_{3}^{-}\right|\right)}{\rho-\rho^{\prime}}\right)^{\left(k-\left|\beta_{2}^{+}\right|+\left|\beta_{2}^{-}\right|-\left|\beta_{3}^{-}\right|\right) / 2} \\
& \times C\left(\frac{C\left(l-k-\left|\gamma^{\prime}\right|+\left|\beta_{2}^{+}\right|-\left|\beta_{2}^{-}\right|+\left|\beta_{3}^{-}\right|\right)}{\rho_{0}-\rho}\right)^{\left(l-k-\left|\gamma^{\prime}\right|+\left|\beta_{2}^{+}\right|-\left|\beta_{2}^{-}\right|+\left|\beta_{3}^{-}\right|\right) / 2}\left(\frac{1}{\rho^{\prime}}\right)^{\left|\gamma^{\prime}\right|-\left|\beta_{2}^{+}\right|-\left|\beta_{3}^{-}\right|} .
\end{aligned}
$$

Here we have used the inequality

$$
\frac{\gamma_{+} !}{\left(\gamma_{+}-\beta_{2}^{-}\right) !}\left(\frac{1}{\rho}\right)^{\left|\gamma_{+}\right|} \leq\left(\frac{\left|\beta_{2}^{-}\right|}{\rho-\rho^{\prime}}\right)^{\left|\beta_{2}^{-}\right|}\left(\frac{1}{\rho^{\prime}}\right)^{\left|\gamma_{+}\right|-\left|\beta_{2}^{-}\right|} \quad \text { for } \rho^{\prime}<\rho .
$$

Taking $\rho$ to satisfy

$$
\frac{l-\left|\gamma^{\prime}\right|}{\rho_{0}-\rho^{\prime}}=\frac{l-\left|\gamma^{\prime}\right|-k+\left|\beta_{2}^{+}\right|-\left|\beta_{2}^{-}\right|+\left|\beta_{3}^{-}\right|}{\rho_{0}-\rho}
$$

this can be estimated by

$$
C_{0}^{2} C\left(\frac{C\left(l-\left|\gamma^{\prime}\right|\right)}{\rho_{0}-\rho^{\prime}}\right)^{\left(l-\left|\gamma^{\prime}\right|\right) / 2}\left(\frac{1}{\rho^{\prime}}\right)^{\left|\gamma^{\prime}\right|}\left(\frac{C_{0} M_{0}}{C}\right)^{\left(k-\left|\beta_{2}^{+}\right|+\left|\beta_{2}^{-}\right|-\left|\beta_{3}^{-}\right|\right) / 2} \rho_{0}^{\left|\beta_{2}^{+}\right|+\left|\beta_{3}^{-}\right|} .
$$


(Note that if $\left|\beta_{2}^{+}\right|+\left|\beta_{3}^{-}\right|=k$ then we can take $\rho=\rho^{\prime}$ from the beginning.) If $\rho_{0}$ and $C$ have been chosen as

$$
\rho_{0}=\sqrt{\frac{C_{0} M_{0}}{C}} \leq \frac{1}{18 C_{0}^{2} \sqrt{n+1}(2 d+1)}
$$

then the sum (4.18) brings to $Q_{l}^{\mathrm{I}}$ the term $Q_{l, \gamma^{\prime}}^{\mathrm{I}} D_{t}^{\gamma_{+}^{\prime}} t^{\gamma_{-}^{\prime}}$ such that

$$
\left\|Q_{l, \gamma^{\prime}}^{\mathrm{I}}\right\|_{\mathcal{B}_{\rho^{\prime}}^{\left(\left(-l-\left|\gamma_{+}^{\prime}\right|+\left|\gamma_{-}^{\prime}\right|\right) / 2\right)}} \leq \frac{1}{3} C\left(\frac{C\left(l-\left|\gamma^{\prime}\right|\right)}{\rho_{0}-\rho^{\prime}}\right)^{\left(l-\left|\gamma^{\prime}\right|\right) / 2}\left(\frac{1}{\rho^{\prime}}\right)^{\left|\gamma^{\prime}\right|} .
$$

For $Q_{l}^{\mathrm{II}}$, we have

$$
Q_{l}^{\mathrm{II}}=\sum_{|\gamma| \leq l-1} \sum_{k=1}^{l-|\gamma|} \sum_{2|\alpha|=k} \frac{1}{\alpha !} H_{0}^{+} \partial_{\eta}^{\alpha} H_{0}^{+*} D_{y}^{\alpha} Q_{l-k, \gamma} D_{t}^{\gamma_{+}} t^{\gamma_{-}} .
$$

Hence, for $\rho_{k} \in\left(\rho^{\prime}, \rho_{0}\right)$,

$$
\begin{aligned}
\left\|Q_{l, \gamma}^{\mathrm{II}}\right\|_{\mathcal{B}_{\rho^{\prime}}^{(()-l-|\gamma+|+|\gamma-|) / 2)}} & \leq \sum_{k=1}^{l-|\gamma|} \sum_{2|\alpha|=k} \frac{1}{\alpha !}\left\|H_{0}^{+}\right\|_{\mathcal{H}_{\rho^{\prime}}^{+,(0)}}\left\|\partial_{\eta}^{\alpha} H_{0}^{+* *}\right\|_{\mathcal{H}_{\rho^{\prime}}^{-*,(-|\alpha|)}}\left\|D_{y}^{\alpha} Q_{l-k, \gamma}\right\|_{\mathcal{B}_{\rho^{\prime}}^{((-l+k-|\gamma+|+|\gamma-|) / 2)}} \\
& \leq \sum_{k=1}^{l-|\gamma|}(n+1)^{\frac{k}{2}} C_{0}^{2} C_{0}^{\frac{k}{2}}\left(\frac{M_{0} k}{\rho_{k}-\rho^{\prime}}\right)^{k / 2} C\left(\frac{C(l-k-|\gamma|)}{\rho_{0}-\rho_{k}}\right)^{(l-k-|\gamma|) / 2}\left(\frac{1}{\rho_{k}}\right)^{|\gamma|} \\
& \leq \sum_{k=1}^{l-|\gamma|} C_{0}^{2} C\left(\frac{(n+1) C_{0} M_{0} k}{\rho_{k}-\rho^{\prime}}\right)^{k / 2}\left(\frac{C(l-k-|\gamma|)}{\rho_{0}-\rho_{k}}\right)^{(l-k-|\gamma|) / 2}\left(\frac{1}{\rho_{k}}\right)^{|\gamma|} .
\end{aligned}
$$

If we choose $\rho_{k}$ to satisfy

$$
\frac{l-k-|\gamma|}{\rho_{0}-\rho_{k}}=\frac{l-|\gamma|}{\rho_{0}-\rho^{\prime}}
$$

then the sum can be estimated by

$$
\begin{aligned}
C_{0}^{2} C\left(\frac{C(l-|\gamma|)}{\rho_{0}-\rho^{\prime}}\right)^{(l-|\gamma|) / 2}\left(\frac{1}{\rho^{\prime}}\right)^{|\gamma|} \sum_{k=1}^{l-|\gamma|}\left(\frac{(n+1) C_{0} M_{0}}{C}\right)^{k / 2} & \\
& \leq \frac{1}{3} C\left(\frac{C(l-|\gamma|)}{\rho_{0}-\rho^{\prime}}\right)^{(l-|\gamma|) / 2}\left(\frac{1}{\rho^{\prime}}\right)^{|\gamma|}
\end{aligned}
$$

provided $C \geq 36(n+1) C_{0}^{5} M_{0}$.

The sum in $Q_{l}^{\mathrm{III}}$ can be estimated in the same way as $Q_{l}^{\mathrm{II}}$ and we obtain (4.14) at order $j=l$.

Now we suppose that (4.14)-(4.17) have been established for all $j$. Then we can realize $E=\sum_{j=0}^{\infty} E_{j}$ as follows: For a $\rho<\rho_{0}$ we set

$$
\begin{aligned}
& V_{\rho}=\left\{(t, y, \tau, \eta) \in T^{*}\left(\mathbf{R}^{N}\right) ;|t|<\rho,|y-\grave{y}|<\rho,|\tau /| \eta||<\rho,|\eta /| \eta|-\grave{\eta}|<\rho\right\}, \\
& W_{\rho}=\iota^{-1}\left(V_{\rho}\right)=\left\{(y, \eta) \in T^{*}\left(\mathbf{R}^{n}\right) ;|y-\grave{y}|<\rho,|\eta /| \eta|-\grave{\eta}|<\rho\right\} .
\end{aligned}
$$


By Lemma 3.9, $Q_{l, \gamma} \in \mathcal{B}_{\rho}^{\left(-l / 2-\left|\gamma_{+}\right| / 2+\left|\gamma_{-}\right| / 2\right)}$ has the symbol $b_{l, \gamma}(t, \tau, y, \eta)$ of order $\left(-l-\left|\gamma_{+}\right|+\left|\gamma_{-}\right|\right) / 2$ of type $(1 / 2,1 / 2)$. The estimate (4.14) implies that

$$
\sum_{l-|\gamma|=k} b_{l, \gamma}(t, y, \tau, \eta) \tau^{\gamma_{+}} s^{\gamma_{-}}
$$

converges for $(t, s, y, \tau, \eta) \in \mathbf{R}^{d} \times V_{\rho}$ and that if we set

$$
q(t, s, y, \tau, \eta)=\sum_{k=0}^{\infty} \chi_{k+1}(\eta / \lambda)\left(\sum_{l-|\gamma|=k} b_{l, \gamma}(t, y, \tau, \eta) \tau^{\gamma_{+}} s^{\gamma_{-}}\right)
$$

then, for a sufficiently large $\lambda$, we have $q \in a-S_{1 / 2,1 / 2}^{0}\left(\mathbf{R}^{d} \times V_{\rho}\right)$, where $\chi$ is the function introduced in Section 2.1. The operator

$$
Q=\operatorname{op}(q)_{\stackrel{\circ}{*}^{*}}: \mathcal{C}_{\mathbf{R}^{N}}^{f}\left(x^{*}\right) \rightarrow \mathcal{C}_{\mathbf{R}^{N}}^{f}\left(x^{*}\right)
$$

is well defined through the kernel

$$
Q(t, y, s, z)=(2 \pi)^{-N} \int_{\mathbf{R}^{N}} e^{i(t-s) \tau+i(y-z) \eta} q(t, s, y, \tau, \eta) g(\tau, \eta) d \tau d \eta
$$

where $g$ is a suitable cut-off function of Métivier (see Section 2.1).

If $H_{j, \gamma}^{-*}$ 's satisfy $(4.15)$ then we have $\sum_{j=0}^{\infty} H_{j}\left(y^{*}\right) \in S_{\mathrm{phg}}^{0,1 / 2}\left(\widetilde{\omega}_{\rho} ; \mathcal{H}_{\rho}^{-*}\right)$. In fact, by Lemma 3.12, (4.15) implies $H_{j, \gamma}^{-*}\left(y^{*}\right) D_{t}^{\gamma_{+}} t^{\gamma_{-}}$is in $\mathcal{O}^{(-j / 2)}\left(\widetilde{\omega}_{\rho} ; \mathcal{H}_{\rho}^{-*}\right)$ and, taking $\rho_{\gamma}=$ $\rho+(|\gamma| / j)\left(\rho_{0}-\rho\right)$, we have

$$
\begin{aligned}
\left\|H_{j}^{-*}\right\|_{\mathcal{H}_{\rho}^{-*,(-j / 2)}} & \leq \sum_{|\gamma| \leq j}\left(\frac{M_{0}|\gamma|}{\rho_{\gamma}-\rho}\right)^{|\gamma| / 2} C\left(\frac{C(j-|\gamma|)}{\rho_{0}-\rho_{\gamma}}\right)^{(j-|\gamma|) / 2}\left(\frac{1}{\rho_{\gamma}}\right)^{|\gamma|} \\
& \leq C\left(\frac{C j}{\rho_{0}-\rho}\right)^{j / 2} \sum_{|\gamma| \leq j}\left(\frac{M_{0}}{C \rho^{2}}\right)^{|\gamma| / 2} \\
& \leq C_{\rho}\left(\frac{C_{\rho} j}{\rho_{0}-\rho}\right)^{j / 2} .
\end{aligned}
$$

Let $\left(h_{j, 1}^{-}, \ldots, h_{j, k_{-}}^{-}\right)$be a symbol of $H_{j}^{-*}$. Then $h_{j, l}^{-}$satisfies

$$
\left|\partial_{t}^{\alpha} h(t, y, \eta)\right| \leq C_{\rho} e^{-\rho t^{2}|\eta| / 2}\left(C_{\rho}(j+|\alpha|) /|\eta|\right)^{(j+|\alpha|) / 2}
$$

for $(y, \eta) \in \widetilde{\omega}_{\rho}$ with another constant $C_{\rho}$. This implies, for a sufficiently large $\lambda$,

$$
h_{l}^{-}(t, y, \eta)=\sum_{j=0}^{\infty} \chi_{j+1}(\eta / \lambda) h_{j, l}^{-}(t, y, \eta)
$$

is in $S_{1 / 2,1 / 2}^{0}\left(\mathbf{R}^{d} \times W_{\rho}\right)$ and bounded by $C_{\rho} e^{-\rho t^{2}|\eta| / 2}$. The operator $H_{(l)}^{-*}=\operatorname{op}\left(h_{l}^{-*}\right)$ is now defined through the kernel

$$
H_{(l)}^{-*}(y ; t, z)=(2 \pi)^{-n} \int_{\mathbf{R}^{n}} e^{i(y-z) \eta} \overline{h_{l}^{-}(t, y, \eta)} g(\eta) d \eta
$$

and we have

$$
W F_{A}\left(H_{(l)}^{-*}\right) \subset\left\{(y, 0, y, \eta, 0,-\eta) \in T^{*}\left(R_{y}^{n} \times \mathbf{R}_{t, z}^{N}\right) ; \eta \in \operatorname{supp}(g)\right\} .
$$


Hence, $H^{-*}=\bigoplus_{l} H_{(l)}^{-*}$ is analytic micro-local with respect to $\iota^{-1}$ as desired.

In the same way we can realize $H^{+}$to be analytic micro-local with respect to $\iota$ with symbol in $\left(a-S_{1 / 2,1 / 2}^{0}\left(\mathbf{R}^{d} \times W_{\rho}\right)\right)^{k_{+}}$. Because $\widetilde{\omega}_{\rho}$ is a complex neighborhood of $W_{\rho}$ we write $H^{-*} \in$ op $\left(a-S_{\text {phg }}^{0,1 / 2}\left(W_{\rho} ; \mathcal{H}_{\rho}^{-*}\right)\right)$ and $H^{+} \in$ op $\left(a-S_{\text {phg }}^{0,1 / 2}\left(W_{\rho} ; \mathcal{H}_{\rho}^{+}\right)\right)$for the above realizations of $\sum_{j=0}^{\infty} H_{j}^{-*}$ and $\sum_{j=0}^{\infty} H_{j}^{+}$. Clearly, we can realize $M$ as a matrix of usual pseudo-differential operators on $W_{\rho}$.

Therefore we obtain

THEOREM 4.17. Let

$$
P=\sum_{|\alpha|+|\beta|<m} t^{\alpha} c_{\alpha \beta}\left(x, D_{x}\right) D_{t}^{\beta}
$$

be an operator of order $m / 2$ of the form (2.4) satisfying (2.5). If $m \geq d+1$ then there are $\rho>0$ and operators $H_{0}^{-} \in \operatorname{op}\left(a-S_{\text {phg }}^{0,1 / 2}\left(W_{\rho} ; \mathcal{H}_{\rho}^{-}\right)\right), H_{0}^{+*} \in \operatorname{op}\left(a-S_{\mathrm{phg}}^{0,1 / 2}\left(W_{\rho} ; \mathcal{H}_{\rho}^{+*}\right)\right), Q \in$ $\operatorname{op}\left(a-S_{1 / 2,1 / 2}^{0}\left(\mathbf{R}^{d} \times V_{\rho}\right)\right), H^{+} \in \operatorname{op}\left(a-S_{\mathrm{phg}}^{0,1 / 2}\left(W_{\rho} ; \mathcal{H}_{\rho}^{+}\right)\right), H^{-*} \in \operatorname{op}\left(a-S_{\mathrm{phg}}^{0,1 / 2}\left(W_{\rho} ; \mathcal{H}_{\rho}^{-*}\right)\right)$ and $M \in \operatorname{op}\left(a-S_{\mathrm{phg}}^{0,1 / 2}\left(W_{\rho} ; \mathcal{M}^{ \pm}\right)\right)$such that

$$
\begin{aligned}
& \left(\begin{array}{cc}
P & H_{0}^{-} \\
H_{0}^{+*} & 0
\end{array}\right)\left(\begin{array}{cc}
Q & H^{+} \\
H^{-*} & M
\end{array}\right)=\left(\begin{array}{cc}
\operatorname{Id}_{\mathcal{C}_{\mathrm{R}^{N}}^{f}\left(x^{*}\right)} & 0 \\
0 & \operatorname{Id}_{\left(\mathcal{C}_{\mathbf{R}^{n}}^{f}\left(\stackrel{o}{y}^{*}\right)\right)^{k+}}
\end{array}\right), \\
& \left(\begin{array}{cc}
Q & H^{+} \\
H^{-*} & M
\end{array}\right)\left(\begin{array}{cc}
P & H_{0}^{-} \\
H_{0}^{+*} & 0
\end{array}\right)=\left(\begin{array}{cc}
\operatorname{Id}_{\mathcal{C}_{\mathrm{R}^{N}}^{f}\left(\stackrel{\circ}{x^{*}}\right)} & 0 \\
0 & \operatorname{Id}_{\left(\mathcal{C}_{\mathbf{R}^{n}}^{f}(\stackrel{\circ}{y})\right)^{k-}}
\end{array}\right) .
\end{aligned}
$$

Now Theorem 2.1 is an immediate consequence of this theorem.

5. Examples. In this section we shall present a few simple examples and illustrate how our results are applied to them.

Recall that the parametrix has been constructed through (4.4). Thus we have the following formulas for $M_{j}$ :

$$
\begin{aligned}
M_{0}\left(y^{*}\right)= & -H_{0}^{-*} P_{0} H_{0}^{+} \\
M_{1}\left(y^{*}\right)= & -H_{0}^{-*} P_{1} H_{0}^{+} \\
M_{2}\left(y^{*}\right)= & -H_{0}^{-*} P_{2} H_{0}^{+}+H_{0}^{-*} P_{1} Q_{0} P_{1} H_{0}^{+} \\
& +i\left(H_{0}^{-*}\left\langle\nabla_{\eta} P_{0}, \nabla_{y} H_{0}^{+}\right\rangle+H_{0}^{-*}\left\langle\nabla_{\eta} H_{0}^{-}, \nabla_{y} M_{0}\right\rangle+M_{0}\left\langle\nabla_{\eta} H_{0}^{-*}, \nabla_{y} H_{0}^{+}\right\rangle\right) \\
\vdots & \\
M_{j}\left(y^{*}\right)= & -H_{0}^{-*} P_{j} H_{0}^{+}+F\left(P_{0}, \ldots, P_{j-1}, Q_{0}, H_{0}^{ \pm}, H_{0}^{ \pm *}, M_{0}\right) .
\end{aligned}
$$

Our results are well applicable to operators with double characteristics because in that case the principal operator $\widehat{\sigma}_{\Sigma}(P)$ is transformed into a sum of harmonic oscillators, for which we can get a complete eigenexpansion by means of Hermite functions. So we set for $k=0,1,2, \ldots$,

$$
\psi_{k}(t)=\left(2^{k} k ! \sqrt{\pi}\right)^{-1 / 2}(t-d / d t)^{k} e^{-t^{2} / 2}
$$

so that

$$
\left(-d^{2} / d t^{2}+t^{2}\right) \psi_{k}(t)=(2 k+1) \psi_{k}(t), \quad\left(\psi_{k}, \psi_{l}\right)_{L^{2}(\mathbf{R})}=\delta_{k l} .
$$


EXAMPLE 1. For a non-negative integer $l$, consider the operator

$$
P=D_{t}^{2}+t^{2} D_{y}^{2}-(2 l+1) D_{y}
$$

at $\stackrel{\circ}{x}^{*}=(0 ; d y) \in T^{*}\left(\mathbf{R}^{2}\right)$. Then

$$
Q\left(y^{*}\right)=\sum_{k \neq l} \frac{1}{2(k-l)|\eta|} h_{k}(t, \eta) h_{k}^{*}(t, \eta),
$$

$H_{0}^{ \pm}\left(y^{*}\right)=H^{ \pm}\left(y^{*}\right)=h_{l}(t, \eta)$ and $M\left(y^{*}\right)=0$, where $h_{k}(t, \eta)=|\eta|^{1 / 4} \psi_{k}\left(t|\eta|^{1 / 2}\right)$ for $k=1,2, \ldots$ Hence

$$
H: u(y) \mapsto(2 \pi)^{-1} \int e^{i y \eta} h_{l}(t, \eta) \widehat{u}(\eta) d \eta
$$

gives the isomorphisms

$$
\begin{aligned}
& \mathcal{C}_{\mathbf{R}}^{f}\left(\stackrel{\circ}{y}^{*}\right) \stackrel{\sim}{\rightarrow} \operatorname{Ker}\left(P: \mathcal{C}_{\mathbf{R}^{2}}^{f}\left(\stackrel{\circ}{x}^{*}\right) \rightarrow \mathcal{C}_{\mathbf{R}^{2}}^{f}\left(\stackrel{\leftrightarrow}{x}^{*}\right)\right), \\
& \mathcal{C}_{\mathbf{R}}^{f}\left(\stackrel{(}{y}^{*}\right) \stackrel{\sim}{\rightarrow} \operatorname{Coker}\left(P: \mathcal{C}_{\mathbf{R}^{2}}^{f}\left(\stackrel{\circ}{x}^{*}\right) \rightarrow \mathcal{C}_{\mathbf{R}^{2}}^{f}\left(\stackrel{\ominus}{x}^{*}\right)\right) .
\end{aligned}
$$

In particular, $f(t, y) \in \mathcal{D}^{\prime}\left(\mathbf{R}^{2}\right)$ is in the range of $P$ in a neighborhood of the origin if and only if $H^{*} f(y)$ is micro-analytic at $(0, d y) \in T^{*}(\mathbf{R})$.

Remark. If $l=0$ then $P=\left(D_{t}+i t_{y}\right)\left(D_{t}-i t D_{y}\right)$ and the range of $P$ is equal to the range of $D_{t}+i t D_{y}$. In this case the characterization of the range of $P$ obtained here is equivalent to that of $D_{t}+i t D_{y}$ given by Sato-Kawai-Kashiwara [10]. To see this we

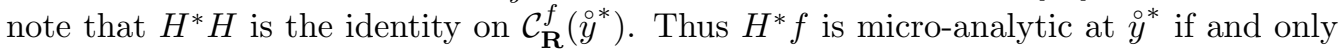
if $H H^{*} f$ is micro-analytic at $\stackrel{\circ}{x}^{*}$. Now $H H^{*}$ has the kernel

$$
(2 \pi)^{-1} \int_{0}^{\infty} e^{i(y-z)-\left(t^{2}+s^{2}\right)|\eta| / 2}|\eta|^{1 / 2} d \eta=\text { const. }\left(y-z+\frac{i}{2}\left(t^{2}+s^{2}\right)+i 0\right)^{-3 / 2},
$$

which is precisely the one appearing in Sato-Kawai-Kashiwara [10, Chap. III, Lemma 2.3.5].

ExAmple 2. Consider

$$
P=D_{t}^{2}+t^{2} D_{y}^{2}-\left(1+t^{k}\right) D_{y}
$$

at $\stackrel{\stackrel{x}{x}}{x}^{*}(0 ; d y) \in T^{*}\left(\mathbf{R}^{2}\right)$. Then $Q_{0}\left(y^{*}\right)=\sum_{l \neq 0} \frac{1}{2 l|\eta|} h_{l}(t, \eta) h_{l}^{*}(t, \eta), H_{0}^{+}\left(y^{*}\right)=H_{0}^{-}\left(y^{*}\right)=$ $h_{0}(t, \eta)$ and we have

$$
\begin{aligned}
& M_{0}\left(y^{*}\right)=\ldots=M_{k-1}\left(y^{*}\right)=0 \\
& M_{k}\left(y^{*}\right)=-h_{0}^{*}(t, \eta) t^{k} \eta h_{0}(t, \eta) \\
& M_{k+1}\left(y^{*}\right)=\ldots=M_{2 k-1}\left(y^{*}\right)=0 \\
& M_{2 k}\left(y^{*}\right)=-\sum_{l \neq 0} \frac{1}{2 l|\eta|}\left|h_{l}^{*}(t, \eta) t^{k} \eta h_{0}(t, \eta)\right|^{2},
\end{aligned}
$$

where $h_{l}(t, \eta)$ are the same as in Example 1.

Hence, $M_{k} \neq 0$ if $k$ is even, $M_{k}=0$ but $M_{2 k} \neq 0$ if $k$ is odd. In both cases we know $\sum_{j=0}^{\infty} M_{j}$ is elliptic at $\stackrel{\circ}{y}^{*}$; therefore $P$ is isomorphic on $\mathcal{C}_{\mathbf{R}^{2}}^{f}\left(\stackrel{\circ}{x}^{*}\right)$.

Example 3. Consider

$$
P=D_{t}^{2}+t^{2}\left(D_{y_{1}}^{2}+D_{y_{2}}^{2}\right)-\left(1-y_{1}^{2}\right) D_{y_{2}}-c
$$


at $\stackrel{\circ}{x}^{*}=\left(0 ; d y_{2}\right) \in T^{*}\left(\mathbf{R}^{3}\right)$ for a $c \in \mathbf{C}$. Then $Q_{0}\left(y^{*}\right)=\sum_{l \neq 0} \frac{1}{2 l|\eta|} h_{l}(t, \eta) h_{l}^{*}(t, \eta)$, $H_{0}^{+}\left(y^{*}\right)=H_{0}^{-}\left(y^{*}\right)=h_{0}(t, \eta)$, where $h_{l}(t, \eta)=|\eta|^{1 / 2} \psi_{l}\left(t|\eta|^{1 / 2}\right), l=1,2, \ldots$, and we have

$$
\begin{aligned}
& M_{0}\left(y^{*}\right)=-\left[\left(\sqrt{\eta_{1}^{2}+\eta_{2}^{2}}-\eta_{2}\right)+y_{1}^{2} \eta_{2}\right], \\
& M_{1}\left(y^{*}\right)=0, \\
& M_{2}\left(y^{*}\right)=c .
\end{aligned}
$$

We note that $\sqrt{\eta_{1}^{2}+\eta_{2}^{2}}-\eta_{2}=\left|\eta_{2}\right|\left(\eta_{1}^{2} /\left(2 \eta_{2}^{2}\right)+O\left(\left|\eta_{1} / \eta_{2}\right|^{4}\right)\right)$ near $\stackrel{\circ}{y}^{*}$. Hence if $c \neq$ $\frac{1}{\sqrt{2}}(2 k+1)$ then $M\left(y, D_{y}\right)$ is isomorphic on $\mathcal{C}_{\mathbf{R}^{2}}^{f}\left(\stackrel{\circ}{y}^{*}\right)$ from the results of Métivier [8]. Otherwise, we can apply Theorem 4.17 once more to $M\left(y, D_{y}\right)$ and find that the reduced operator $\widetilde{M}\left(D_{y_{2}}\right)$ is elliptic at $\left(0 ; d y_{2}\right) \in T^{*}(\mathbf{R})$. Therefore, for all $c \in \mathbf{C}$, we conclude that $P$ is an isomorphism on $\mathcal{C}_{\mathbf{R}^{2}}^{f}\left({ }^{*}\right)$.

ExAmple 4. Consider

$$
P=\sum_{i=1}^{d}\left(D_{t_{i}}^{2}+t_{i}^{2} D_{y}^{2}-D_{y}\right)+\sum a_{i j}(y) t_{i} t_{j} D_{y}\left(=P_{0}+P_{2}\right)
$$

at $\stackrel{\stackrel{x}{ }}{*}^{*}=(0 ; d y) \in T^{*}\left(\mathbf{R}^{d+1}\right)$. We set $A=D_{y}^{-1} P\left(D_{y}^{-1} P_{0}+1\right)^{[d / 2]}$ and apply Theorem 4.17 to $A$. Then $H_{0}^{+}\left(y^{*}\right)=H_{0}^{-}\left(y^{*}\right)=|\eta|^{d / 4} \Pi_{i=1}^{d} \psi_{0}\left(t|\eta|^{1 / 2}\right)$ and we have

$$
\begin{aligned}
M_{0}\left(y^{*}\right) & =M_{1}\left(y^{*}\right)=0, \\
M_{2}\left(y^{*}\right) & =-H_{0}^{-*}\left(y^{*}\right)\left(D_{y}^{-1} P_{2}\left(D_{y}^{-1} P_{0}+1\right)^{[d / 2]}\right) H_{0}^{+}\left(y^{*}\right) \\
& =-H_{0}^{-*}\left(y^{*}\right)\left(\sum a_{i j}(y) t_{i} t_{j}\right) H_{0}^{+}\left(y^{*}\right) \\
& =-\frac{1}{2} \sum_{i=1}^{d} a_{i i}(y) .
\end{aligned}
$$

Here we have used the fact that $P_{0} H_{0}^{+}\left(y^{*}\right)=0$. Hence if $\sum_{i=1}^{d} a_{i i}(0) \neq 0$ then $P$ is isomorphic on $\mathcal{C}_{\mathbf{R}^{d+1}}^{f}\left(\stackrel{\circ}{*}^{*}\right)$.

\section{References}

[1] L. Boutet de Monvel, Hypoelliptic operators with double characteristics and related pseudodifferential operators, Comm. Pure Appl. Math. 27 (1974), 585-639.

[2] L. Boutet de Monvel, A. Grigis et B. Helffer, Paramétrixes d'opérateurs pseudodifférentiels à caractéristiques multiples, Astérisque 34-35 (1976), 93-121.

[3] A. Grigis and L. P. Rothschild, A criterion for analytic hypoellipticity of a class of differential operators with polynomial coefficients, Ann. Math. 118 (1983), 443-460.

[4] V. V. Grushin, On a class of elliptic pseudodifferential operators degenerate on a submanifold, Math. USSR-Sb. 13 (1971), 155-185.

[5] B. Helffer, Sur l'hypoellipticité des opérateurs pseudodifférentiels à caractéristiques multiples (perte de 3/2 dérivées), Bull. Soc. Math. France 51-52 (1977), 13-61.

[6] T. Kato, Perturbation Theory for Linear Operators, Springer, Berlin, 1980. 
[7] M. Kashiwara, T. Kawai and T. Oshima, Structure of cohomology groups whose coefficients are microfunction solution sheaves of systems of pseudo-differential equations with multiple characteristics I, Proc. Japan Acad. 50 (1974), 420-425.

[8] G. Métivier, Analytic hypoellipticity for operators with multiple characteristics, Comm. Partial Differential Equations, 6 (1981), 1-90.

[9] A. Melin, Parametrix constructions for some right invariant operators on the Heisenberg group, ibid., 1363-1405.

[10] M. Sato, T. Kawai and M. Kashiwara, Microfunctions and Pseudo-Differential Operators, Lecture Notes in Math. 287, Springer, 1973, 265-529.

[11] J. Sjöstrand, Parametrix for pseudodifferential operators with multiple characteristics, Ark. Mat. 12 (1974), 85-130.

[12] E. M. Stein, An example on the Heisenberg group related to the Lewy operator, Invent. Math. 69 (1982), 209-216.

[13] F. Treves, Analytic hypo-ellipticity of a class of pseudodifferential operators with double characteristics and applications, Comm. Partial Differential Equations 3 (1978), 475642 .

[14] F. Treves, Introduction to Pseudodifferential and Fourier Integral Operators, Vols. I, II, Plenum Press, New York and London, 1981. 